\title{
Solar Wind Control of Electric Fields and Currents in the Ionosphere
}

\author{
Y. I. FeldSTEIN and A. E. LeVitin \\ IZMIRAN, Troitsk, Moscow Region 142092, U.S.S.R.
}

(Received October 1, 1985; Revised June 12, 1986)

\begin{abstract}
KERN (1966) substantiated theoretically the feasibility of using geomagnetic field variations on the earth's surface to calculate the spatial structure of the current system. Algorithms of the electric field and current calculation being used at present are examined. The methods for discriminating the geomagnetic variations controlled by various solar wind parameters are discussed. The calculation results obtained for the assumption of the uniform ionospheric conductivity are presented. This approximation made it possible to account for the existing regularities in the distribution of field-aligned currents underdefinite solar wind conditions and to predict the character of the three-dimensional current system in the intervals with the positive $B_{z}$ IMF. The methods for and the results of calculating the electrostatic potential, the electric field, the ionospheric and field-aligned currents in the highlatitude ionosphere for nonuniform conductivity are presented in detail. The calculation results are compared with the direct measurements of the respective parameters.
\end{abstract}

\section{Introduction}

The electric and magnetic fields in the Earth's magnetosphere give rise to the large-scale convective motions of plasma. The changes of the motions in space and time which are closely associated with the solar wind create the electromagnetic weather of the near Earth space environment. On the Earth's surface the electromagnetic weather leaves its signature in the geomagnetic field variations which respond keenly to any changes of the electric fields in the Earth's magnetosphere and ionosphere. The geomagnetic field variations at high latitudes (at corrected geomagnetic latitude $\Phi \geq 60^{\circ}$ ) are of special interest because in this zone the remote magnetospheric regions interact directly with the solar wind via magnetic field lines.

A number of international research programs involved in the collection of observational data at the Earth's upper atmosphere and magnetosphere have recently been completed resulting in data bases which are unparalleled in the history of science. Present-day science is faced with the most important and urgent task of systematizing and classifying these data bases and of finding the relationships among the diverse processes they describe.

International Geophysical Year (IGY) data analyses yielded two concepts which facilitated the ordering of the space-time description of the occurrence of geophysical events, namely the concept of the auroral oval (FELDSTEIN et al., 1969) and the concept of the auroral (magnetospheric) substorm (AKASOFU, 1968). The oval 
concept made it possible to order the events observed at high latitudes because the auroral oval is closely associated with large-scale magnetospheric structure and may be used as a natural, spatial coordinate system to describe the processes occurring in the high-latitude, upper atmosphere. The substorm concept facilitated the temporal ordering of the events since the character of the processes in the upper atmosphere and in the magnetosphere is essentially contingent upon the location and the rate of injection and dissipation of solar wind energy inside the magnetosphere.

In recent years, it has become obvious that the occurrence of the high-latitude geophysical events in the Earth's environment is controlled to a great degree by the interplanetary medium parameters, namely, by the direction and intensity of the interplanetary magnetic field (IMF) vector, and by the solar wind plasma velocity and density (AKASOFU and CHAPMAN, 1972; NISHIDA, 1978; LYATSKY, 1978; LYATSKY and MALTSEV, 1983; SERGEeV and TSYGANENKO, 1980). The quantitative relations were established between the electric and magnetic fields and electric currents at altitudes above $100 \mathrm{~km}$ and the interplanetary magnetic fields. As a result, the third concept was formulated, namely, the interplanetary medium parameters control the electromagnetic processes in the high-latitude magnetosphere (the ionosphere included) which give rise to the geophysical events in this region of the Earth's environment. The International Magnetospheric Study Project (IMS) has made it possible to further develop the concept using complex experimental data. Subsequent years saw the construction of the quantitative models of the space-time distributions of the large-scale electric fields and of the ionospheric and field-aligned currents in the auroral and polar cap regions. The most comprehensive models were constructed on the basis of the geomagnetic field variations observed on the Earth's surface. Thus, the long-standing dream of geophysicists became real, namely, the algorithm was developed to infer the three-dimensional current system and the quasi-steady state electromagnetic field of the ionosphere from ground-based geomagnetic data.

The feasibility of using the ground-based magnetic data to obtain the electric fields and currents of the ionosphere has initiated an essentially new and very important phase in the development of the geophysics. Novel numerical methods for solving the equations which relate the magnetic field on the Earth's surface $\boldsymbol{H}$ to the ionospheric electric field potential $\phi$ made it possible to infer the real-time spatial distribution of fields and currents in the entire high-latitude region from the continuous recording of geomagnetic variations at stations in the existing network. At the same time, the vast volume of geomagnetic data accumulated during quite a number of years has made it possible to carry out the statistical studies, namely, to relate the geomagnetic field variations to the interplanetary medium parameters.

In numerous works, the combination of ground-based and space-craft measurements proved to be a powerful tool to study the diverse and complicated events in both space and time. As stressed by VASYLIUNAS (1970, 1972), TVERSKOY (1972), GUREVICH et al. (1975, 1976), and BOSTRÖM (1975), the magnetosphere and the ionosphere must be examined as a whole. This idea was realized by LYATSKY and MALTSEV (1972), and JAGGI and WOLF (1973) and was developed further via extensive, computer simulation models. The numerical simulations of the Rice University group have become a power tool to study the dynamics of the magnetosphere-ionosphere system (HAREL et al., 1981).

In the subsequent sections of this work we shall trace the evolution of the ideas set 
forth by CHAPMAN (1935) and ALFVÉN (1950) concerning the character of the system responsible for the geomagnetic field variations observed in high latitudes. Subsequently KERN (1966) theoretically proved the feasibility of using geomagnetic field variations on the Earth's surface to calculate the spatial structure of the current system for a uniform anisotropic conductivity of the ionosphere. Then, KRYLOV (1974) and GUREVICH et al. (1975) developed methods for the same calculations using nonuniform ionospheric conductivity. We shall also examine the electric field and current calculation algorithms used at present elsewhere. Since the calculations are based on the high-latitude geomagnetic field variation data, we shall briefly discuss the methods for discriminating the geomagnetic variations controlled by various solar wind parameters. The calculation results are compared with the direct measurements of the respective parameters in the near Earth space.

2. Magnetic Field Variations on the Earth's Surface and Associated Current Systems

The spatial distribution of magnetic field vectors on the Earth's surface do not permit an unambiguous definition of the current system which caused a given observed geomagnetic variation. The solution of such a problem requires that definite assumptions to be introduced concerning the current configuration, the conductivity distribution, the ionospheric thickness etc. The current systems most vividly discussed were proposed by Birkeland, Chapman, Vestine, and Alfvén, namely, the ChapmanVestine system (VESTINE and CHAPMAN, 1938) and the Birkeland-Alfvén system (BIRKELAND, 1913; ALFVÉN, 1950). Later on, the magnetic field variations at the Earth's surface were explained using both pure ionospheric and three-dimensional current systems (KIRKPATRICK, 1952; BOSTRÖM, 1964; AKASOFU and MENG, 1969).

The unambiguous experimental evidence for the existence of the field-aligned currents above the ionosphere was obtained by analyzing the satellite magnetic measurements at altitudes of $\sim 1000 \mathrm{~km}$ (ZMUDA and ARMSTRONG, 1974 and the reviews by POTEMRA, 1983; AKASOFU, 1984; FELDSTEIN et al., 1982a; SAFLEKOS et al., 1982). The measurements were compared against simultaneous theoretical calculations which showed that, for uniform ionospheric conductivity and radial magnetic field lines along which the field-aligned current, $j_{\|}$, is streaming, the fieldaligned current-produced magnetic signal on the Earth's surface is completely cancelled by the magnetic field of the ionospheric Pedersen currents (FUKUSHIMA, 1976). This theorem is satisfied for both planar and spheric ionospheres and for any distribution of the currents streaming to and from the ionosphere.

Fukushima's works impelled most of the researchers to conclude that the real three-dimensional current system in the high-latitude ionosphere cannot in principle be calculated using ground-based magnetic data only. This opinion prevailed despite KERN (1966) where he proposed a scheme to compute the spatial distribution of the ionospheric and field-aligned currents using values of the magnetic field $\boldsymbol{H}$ on the Earth's surface. This situation prevailed until MISHIN and POPOV, 1969) used Kern's relation to calculate the values of $j_{\|}$from the magnetic field of the $S D$-variation in May-August, 1958. Figure 1 shows the field-aligned current isolines obtained by Mishin and Popov for the northern (top) and southern (bottom) hemispheres. Current flowing into the ionosphere is drawn with the solid lines, and currents from 

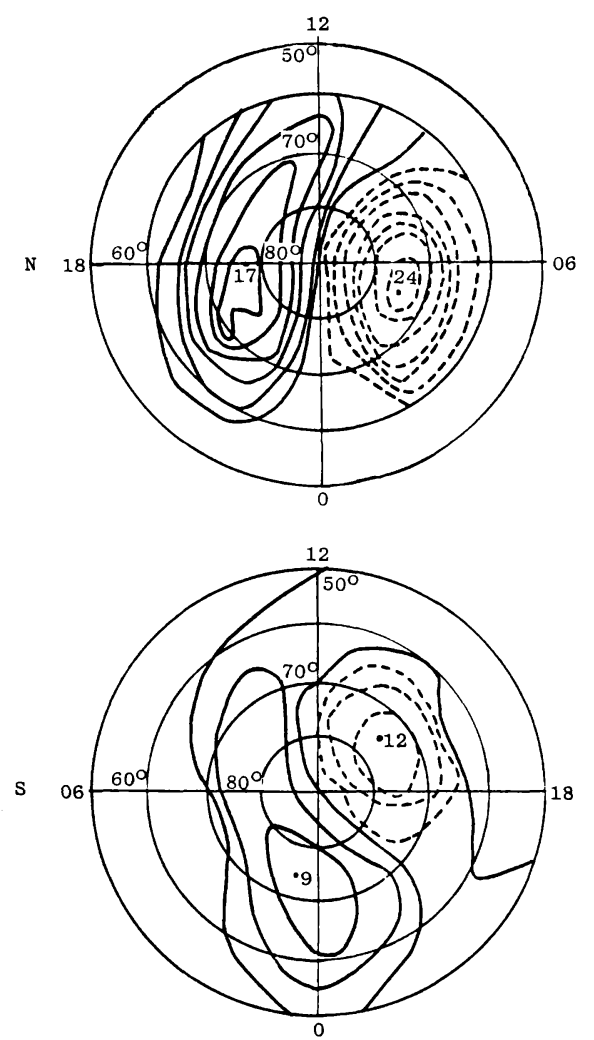

Fig. 1. The distribution of $j_{\|}$in the northern (above) and southern (below) hemispheres inferred from the data from the disturbed solar-diurnal geomagnetic field variation $S D$ in May-August, 1958 (MISHIN and PoPOV, 1969). The dashed lines show the field-aligned current flowing out of the ionosphere, the solid lines show the field-aligned current flowing into the ionosphere. The contour interval is $3.2 \times 10^{-7}$ $\mathrm{A} / \mathrm{m}^{2}$.

the ionosphere with the dashed lines. Their criticism of the Kern formula was most probably motivated by the strange patterns in the spatial distribution of $j_{\|}$that they obtained. Present-day knowledge of large-scale field-aligned currents during magnetic disturbances (IMF $B_{z}<0$ ), does not agree with the $j_{\|}$distribution shown in Fig. 1. We must note here that some scientists still have doubts as to the validity of the methods for inferring three-dimensional current systems from the ground-based magnetic data only.

For simple models of the nonuniform ionospheric conductivity, the relationship between the equivalent and field-aligned currents was represented by analytical expressions by LYATSKY and MALTSEV (1972). The theory for simulating the electric fields and currents in the high-latitude ionosphere from ground-based geomagnetic data was developed in a series of works (the relevant references may be found in KRYLOV, 1974 and GUREVICH et al., 1975, 1976). The authors have derived the equation for the electric field potential $\phi$ in the Earth's magnetosphere and ionosphere. For non-uniform ionospheric conductivity, the equation must be solved by numerical methods. FAERMARK (1980) described the difference method calcula- 
tion procedure to infer the electric fields and currents in the latitudinal zone $\geqslant 60^{\circ}$ from the geomagnetic variations obtained by AFONINA et al. (1980) and LEVITIN et al. (1982). The calculations are made in terms of a particular model of the conductivity of the high-latitude ionosphere.

MATVEEV and SHPYNEV (1975) following Kern's method developed an equation for computing electrostatic potential which is analogous to that obtained by GUREVICH et al. (1975) and allows for nonuniform ionospheric conductivity. The solution was aimed at the analytical representation of the geomagnetic variation field from a confined, nonuniformly-distributed network of stations (MISHIN et al., 1977). The scheme of the solution was as follows: 1) The current function $\Psi$ is determined by selecting the optimal spectrum of spherical harmonics for the initial magnetic variation field. 2) The magnetic and electrostatic potentials are represented by a spherical harmonic expansion. 3) $N$ linear equations for the potential expansion coefficients are formed on the set of $N$ points at which the conductivity, its derivative, and the current function are given. A similar method was used by MISHIN et al. (1980; 1981 and 1982 and the references therein) to infer the electric fields and currents in the high-latitude ionosphere from ground-based geomagnetic data.

In the late seventies, KISABETH (1979) developed a different method for finding the parameters of three-dimensional current systems from the data of a chain of geomagnetic stations (see also KISABETH and ROSTOKER, 1977). The method does not solve the equation for the potential, rather it selects ionospheric current intensities which permit the best convergence of the calculated magnetic disturbance produced by a given current system with the real magnetic signal detected by a meridional chain.

KAMIDE et al. (1981) presented the height-integrated ionospheric current to be a sum of the toroidal and poloidal currents related to the equivalent current function $\Psi$ (see also KERN, 1966). The logic of the inference of fields and currents from groundbased geomagnetic data is as follows: 1st obtain the initial magnetic field variations using the data from a network of high-latitude observatories; 2nd determine the potential of the initial magnetic field $(H=-\nabla U)$ and the current function $\Psi$; 3rd select a conductivity model $\left(\Sigma_{\mathrm{H}}, \Sigma_{\mathrm{P}}\right)$; 4 th numerically solve the equation for electrostatic potential $(E=-\operatorname{grad} \phi)$ by the difference method on the network with a latitude step $1^{\circ}$ and a longitude step $\sim 15^{\circ}$; 5th compute the horizontal current distribution; and 6th compute $j_{\|}$as the divergence of the horizontal current. Numerical procedures are described in more detail by KAMIDE and KROEHL (1984).

Thus, independently of each other, several groups of researchers have developed and applied the calculational methods which make it possible to infer the distribution of the potential $\phi$, the electric field $E$, the horizontal ionospheric current $j_{\perp}$, and the field-aligned current $j_{\|}$from the variation of the geomagnetic field $\boldsymbol{H}$ on the Earth's surface. In their calculations, each of the groups used a particular conductivity model, a particular data base for the magnetic field variations, and a particular method of numerical calculations.

3. The High-Latitude Geomagnetic Variation Field Structure Relevant to the Solar Wind Parameters

Both magnetospheric substorm fields and the magnetic field variations at high latitudes closely related to the parameters of the solar wind and of the frozen IMF 
(AKASOFU, 1977, 1981; NISHIDA, 1978, 1983; BOSTRÖM, 1975; FRIIS-CHRISTENSEN, 1981; AlfVÉn, 1977, 1981; FeldSTeIN, 1976; TroshicheV, 1982; KAMIDE, 1982, 1984; STERN, 1983; SAFLEKOS et al., 1982). The variations involve the entire highlatitude region from the auroral zone to the pole and are observed continuously during quiet and disturbed periods. The DP2 variations relevant to the $B_{z}$ IMF component and described first by NISHIDA $(1968 \mathrm{a}, \mathrm{b})$ are the characteristic example of such geomagnetic field variations. SVALGAARD (1968) and MANSUROV (1969) noted the characteristic field variations relevant to the IMF sector structure. Later on, FRIIS-CHRISTENSEN et al. (1972), SUMARUK and FELDSTEIN (1973), and KAWASAKI et al. (1973) showed that these variations were controlled by the $B_{y}$ component. Based on the close relationships of all three geomagnetic field components in the near-pole region to the $B_{y}$ component, FELDSTEIN et al. (1975) and FRIIS-CHRISTENSEN and WILHJELM (1975) proposed a regression method for extracting the $B_{y}$ componentcontrolled fraction of the high-latitude magnetic field variation. The method was used later by MAEZAWA (1976) for the $B_{z}$ component. The method was then used by MISHIN (1978) and AFONINA et al. (1979) to find the geomagnetic field variation fraction controlled by solar wind velocity, $V$, and density, $n$. The near-pole magnetic field variations controlled by the positive $B_{z}$ values were identified by IWASAKI (1971), MAEZAWA (1976), KuZNETSOV and TROSHICHEV (1977), and others.

The regression analysis method permits the magnetic field components on the Earth's surface to be related quantitatively to the solar wind plasma parameters and to the IMF components. The analysis was carried out by several groups with different sets of parameters. For example, LEVITIN et al. (1982) have presented hourly means of the horizontal and vertical components of the field variations on the Earth's surface in the $\Phi \geqslant 60^{\circ}$ latitude region to be the series

$$
H_{E}^{i}=K_{i}^{B_{y}} B_{y}+K_{i}^{B_{i}} B_{z}+K_{i}^{B_{x}} B_{x}+K_{i}^{n V^{2}} n\left(\frac{V}{100}\right)^{2}+K_{i}^{V^{2}}\left(\frac{V}{100}\right)^{2}+K_{i}^{\left|B_{y}\right|}\left|B_{y}\right|+H_{0}^{i}
$$

The series is assumed to be sufficient when describing the features of the geomagnetic field variations for the quasistationary processes in the Earth's magnetosphere as related to the interplanetary medium conditions. The addition of new terms fails to improve the agreement between the observed and model-calculated values of $H_{E}^{i}$. The coefficients $K_{i}$ are found by the least squares method from the condition of the best description of the disturbed field through the IMF components and the interplanetary parameters. Each of the series terms is a geomagnetic field response to a certain interplanetary medium parameter, while the residual term $H_{0}^{i}$ is the extrapolation of the mid-latitude $S_{q}$ current system to the high-latitude zone. The determination of the coefficients, $K_{i}$, by the least squares method for each UT hour and for each observatory has been described in detail by MAEZAWA (1976), TROSHICHEV and TSYGANENKO (1979), and LEVITIN et al. (1982). The coefficients $K_{i}^{B_{x}}$ and $K_{i}^{\left\langle B_{y}\right\rangle}$ are much smaller than $K_{i}^{B_{y}}$ and $K_{i}^{B_{z}}$, while the terms depending on $n$ and $V$ may be combined with $H_{0}^{i}$ to constitute a single constant, $H_{0}=K_{i}^{n V^{2}}$ $n(V / 100)^{2}+K_{i}^{V^{2}}(V / 100)^{2}+H_{0}^{i}$, which characterizes the mean conditions in solar wind ( $n=4$ particles $/ \mathrm{cm}^{3}$ and $V=500 \mathrm{~km} / \mathrm{s}$ from LEVITIN et al., 1982). Thus, if the magnetic field variations are presented as a series in solar wind parameters, the mean values of the solar wind plasma parameters may be limited to the trinomial 


$$
H_{E}^{i}=K_{i}^{B_{y}} B_{y}+K_{i}^{B_{z}} B_{z}+H_{0}
$$

The representation (1) has to be used in case of the extremal values of $n$ and $V$.

The series-expansion (2) of the hourly means of the high-latitude field variation components was used by MAEZAWA (1976), BELOV et al. (1977), TROSHICHEV and TSYGANENKO (1979), and TROSHICHEV et al. (1979). A similar expansion was made by FRIIS-CHRISTENSEN (1979) using the 20-min means of the field components from the Greenland meridional chain of magnetic observatories. Two sets of the coefficients $K_{i}$ were derived for the $B_{z}<0$ and $B_{z}>0$ data sets, respectively. The use of the hourly or 20-min means of the field components and the fairly simple $H_{E}^{i}$ representation in terms of a series of the interplanetary medium parameters have permitted the description of only the quasisteady-state geomagnetic field variations. A somewhat different approach has to be used in describing the rapid and complicated magnetic field variations during magnetospheric substorms which are closely related to the intrinsic magnetospheric processes giving rise to the local changes of the current system geometry. For example, the mean error in calculating $H_{E}^{i}$ from the relation (2) was estimated (LEVITIN et al., 1982) to be $\sim 30 \mathrm{nT}$, in polar cap and $\sim 50 \mathrm{nT}$ in auroral zone. The substorm-time disagreement may rise to $\sim 100$ $\mathrm{nT}$ because the applied methods for separating the high-latitude geomagnetic field variations relevant to the interplanetary medium parameters make it possible to describe only the fairly smooth geomagnetic field fluctuations related directly to the interplanetary medium parameters. Another and more original approach to describing the pulsed field variations during substorms seems to be necessary.

The equivalent currents used below have been obtained (BELOV et al., 1977; LEVITIN et al., 1982) by the regression analysis method and are due to a 1-nT change of the $B_{y}, B_{z}<0, B_{z}>0$ components and in case of $B_{z}=B_{y}=0$.

MISHIN et al. (1978, 1982 and the references therein) made use of another method for finding the on-surface geomagnetic field variations controlled by the IMF components and by the solar wind parameters. The magnetic disturbance potential, $U$, on the Earth's surface is presented to be the series

$$
U(\Theta, \Lambda)=R_{\mathrm{E}} \sum_{k=1}^{\infty} d_{k} G_{k}(\Theta, \Lambda)
$$

where $G_{k}$ is the eigenfunction of the Laplace equation. The coefficients $d_{k}$ are calculated using the measured values of the $X, Y, Z$-components of the geomagnetic field vector from the network of stations $\left\{\Theta_{i}, \Lambda_{i}\right\}(i=1, \ldots, N)$. The current function, $\Psi_{e}$, which describes the equivalent ionospheric current, is determined through the same coefficients $d_{k}$. The finite number of observation points restricts the length of the series (3). Therefore, the "optimal spectrum" (definite values of $K$ ) is selected by estimating the errors in the coefficients of the spherical series. Such a selection is made using the orthogonalization of the basis function on the chosen set of reference points.

After that, the coefficients, $d_{k}$, are subjected to regression analysis whose regression series contains terms comprising the solar wind parameters, the universal time (UT), and the geomagnetic activity indices. The length of the series is varied and may include up to 40 terms, namely, $b=$ const, $V, V^{2}, V^{3}, V \cos (\mathrm{UT}), V \sin (\mathrm{UT}), n, n^{2}$, $n^{1 / 3} V, n V, n V^{2}, B_{y}, B_{y}^{2}, B_{y}^{3}, B_{y} V, B_{y}^{2} V, B_{y} V^{2}, B_{z}, B_{z}^{2}, B_{z}^{3}, B_{z} V, B_{z}^{2} V^{2}, B_{z} V^{2}, b \cos (\mathrm{UT})$, $b \sin (\mathrm{UT}), b \cos (2 \mathrm{UT}), b \sin (2 \mathrm{UT}), A E, A E^{2}, B_{y} B_{z}, B_{y}^{2} B_{z}, B_{y} B_{z}^{2}, n \cos (\mathrm{UT}), n \sin (\mathrm{UT})$, 
$B_{y} \cos (\mathrm{UT}), B_{y} \sin (\mathrm{UT}), B_{z} \cos (\mathrm{UT}), B_{z} \sin (\mathrm{UT})$, etc.

The reference points $\left\{\Theta_{i}, \Lambda_{i}\right\}$, whose data are used to determine $d_{k}$, amount to about 100 , while the time interval of data sampling is some 10 days. The night-time values of the $X, Y$ and $Z$-components of the geomagnetic field vector (quiet geomagnetic conditions) are taken to be the reference level for $X, Y$ and $Z$ variations.

The drawbacks of the analysis method described above are (i) the necessity for the reference level of the geomagnetic variation field to be introduced before making the correlation analysis and the associate possible distortion of the real current systems controlled by the solar wind parameters; (ii) the application of the spherical harmonic analysis in the high-latitude zone, especially in polar cap, where the magnetic observatories are rare and distributed nonuniformly (there are less than ten of them in polar cap), while geomagnetic variations are local. As a result, the very high harmonics of the expansion are inferred from the observation point data which are not very sensitive to the variations localized in the polar cap; (iii) a great number of arguments in the regression equation relating $d_{k}$ to the solar wind parameters. As a result, the model gets tedious, the expansion terms depending on each other appear, and the "elementary" geomagnetic variations controlled by $B_{y}$ and $B_{z}$ components become impossible to separate; (iv) a low reliability of the results because they were obtained for small sample size without grouping into the $B_{z}>0$ and $B_{z}<0$ IMF time intervals.

BAZARZHAPOV et al. (1983) have also employed the regression analysis method in a manner similar to that used by MAEZAWA (1976), BELOV et al. (1977), FRIISChristensen (1979), and Troshichev and Tsyganenko (1979). They have obtained a geomagnetic variation model based on the regression equations relating the hourly means of the $X, Y, Z$-components of the geomagnetic field vector at given stations to the solar wind parameters.

4. Can the Three-Dimensional Current System Be Inferred from the Geomagnetic Field Variations on the Earth's Surface?

In Section 2 we discussed the algorithms proposed by various groups for restoring the character of the current system responsible for the geomagnetic field variations observed on the Earth's surface. The natural question arises: what is the extent to which the results obtained are correct? The question may be answered in only one way, namely, by comparing the model calculation results with direct spaceborn measurements, either instantaneously at a given point in time or averaged to obtain a global picture, or by comparison with radar data (LEVITIN et al., 1977a, b; FELDSTEIN et al., 1982b, c, 1984a; BAKER and KAMIDE, 1985).

KAMIDE et al. (1982) and WOLF and KAMIDE (1983) tested the representativity of the method for calculating the magnetospheric fields and currents from groundbased magnetic data. They used a two-step procedure in which (1) the magnetic signal at the Earth's surface was calculated for given electromagnetic conditions in the magnetosphere and (2) the distributions of the magnetospheric fields and currents were calculated from a magnetic disturbance on the Earth's surface using an appropriate algorithm, whereupon the distributions obtained were compared. However, the attempts were aimed rather at verifying the correctness of the mathematical formalism involved than at testing the physical assumptions used to 
restore the magnetospheric fields and currents from the geomagnetic variations at the Earth's surface.

By 1976-1977, when FAERMARK et al. (1976) and LEVITIN et al. (1977a, b) started using the ground-based magnetic data to restore the current system in the Earth's magnetosphere, the space-time distribution of the large-scale field-aligned currents had been safely inferred from the observations on board the OGO, TRIAD, and ISIS-2 satellites (SUGIURA, 1975; ZMUDA and ARMSTRONG, 1974; IIJIMA and Potemra, 1976a, b; Potemra, 1977; Burrows et al., 1976; MCDiARmid et al., 1977). The corrected geomagnetic latitude-local geomagnetic time coordinates are used in Fig. 2(a)-(d) to present several schemes of the field-aligned current distribution inferred from the Triad observations. The disturbances transverse to the force lines of the main geomagnetic field, and hence the field-aligned currents, exist at any time of a day and at any magnetic activity level. The field-aligned currents flow into and out of the ionosphere at auroral latitudes. During the 1400-2300 (0000-1000) LT interval, the field-aligned current is inward (outward) on the equatorward side of the oval and is outward (inward) on the poleward side of the auroral oval. According to ZMUDA and ARMSTRONG (1974), magnetic disturbances are observed continuously from 1000 to $1400 \mathrm{LT}$ and do not involve any definite characteristic direction of the field-aligned currents (Fig. 2(a)). According to IIJIMA and POTEMRA (1976b), however, field-aligned current zone 3 is located poleward of zone 1 during magnetically quiet periods (Fig. 2(b)). In contrast to zone 1, the zone 3 field-aligned current flows into the ionosphere in the afternoon sector and out in the prenoon sector, but the chaotic direction of $j_{\|}$is preserved near noon (the oblique shading in Fig. 2(b)). Zone 3 is associated with the dayside cusp, the $j_{\|}$intensity in that zone increases with enhanced the southward $B_{z}$ component and is independent of the $B_{y}$ component. The character of the relationships of $j_{\|}$to the IMF components in zone 3 was established by IIJIMA and POTEMRA (1976b) using the limited data of 11 orbits of the satellite. However, the pre- and afternoon traversals of the dayside cusp were not separated and the intensities and signs of the $B_{y}$ component during the traversals were not indicated. In this model, the field-aligned current zones 1 and 2 exist during both quiet and disturbed periods, while zone 3 disappears during disturbed periods (Fig. 2(c)). The polar cap is characterized by small-scale transverse disturbances of the geomagnetic field (Fig. 2(d)) which are most frequently ( $>75 \%$ of the cases) detected in the dawn sector from 0300 to $0900 \mathrm{MLT}$ for $75^{\circ}<\Phi<82^{\circ}$. In the north polar cap, the disturbances are more frequent when the IMF is antisunward than in case of the sunward IMF. During the periods of persistent and intensive $B_{z}>0$, the large-scale field-aligned currents at auroral oval latitudes degenerate into small-scale currents.

Following LEVITIN et al. (1977a, 1982), Figures 3(a)-(d) present the results of the model calculations of the space-time distributions of $j \|$ inferred from the magnetic field variation data. The height-integrated ionospheric conductivity was assumed to be uniform, thereby permitting $j \|$ to be calculated from the simple relation

$$
j_{\|}^{M}\left(\mathrm{~A} / \mathrm{km}^{2}\right)=\frac{10}{4 \pi} \frac{\Sigma_{\mathrm{p}}}{\Sigma_{\mathrm{H}}} \operatorname{div} H(\mathrm{nT} / \mathrm{km}) .
$$

The allowance for the nonuniform ionospheric conductivity in the real summer conditions does not in practice effect the character of the space-time distribution of $j_{\|}$. 


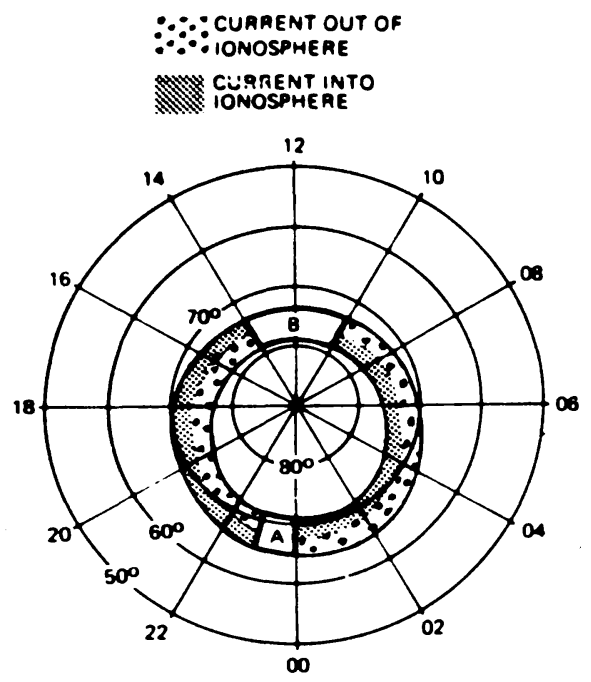

a

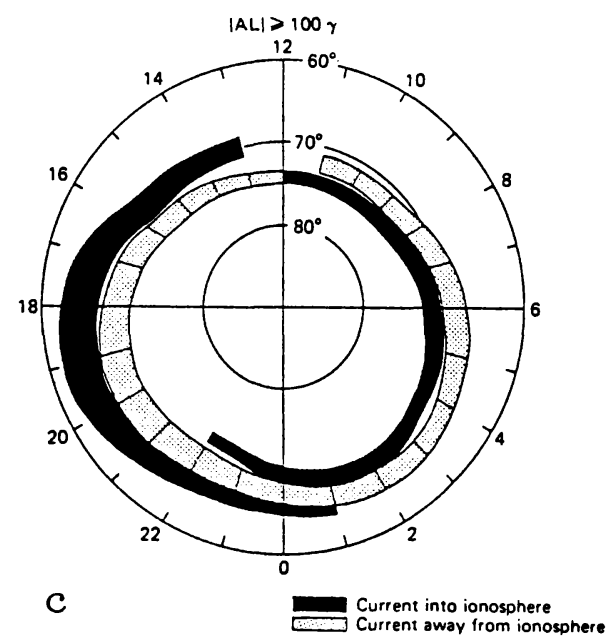

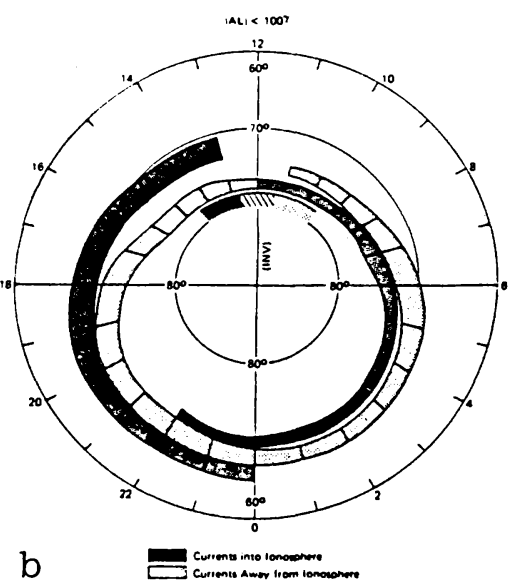

Frequency of occurrence distribution of transverse variations for all $\mathrm{KD}$

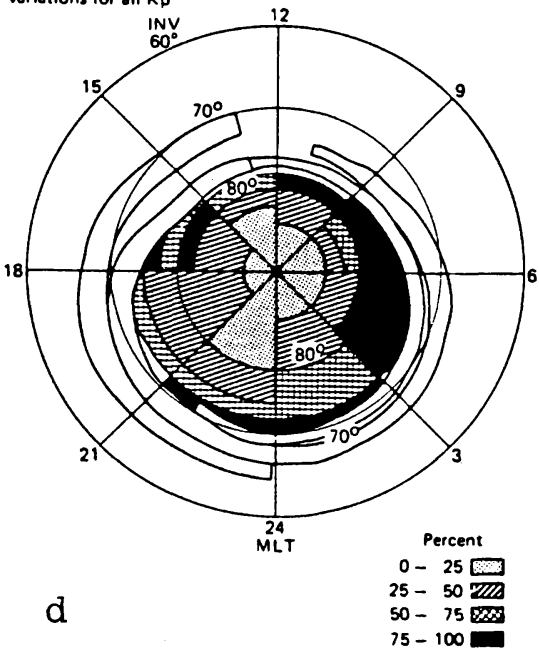

Fig. 2. The space-time distribution of $j_{\|}$inferred from TRIAD data. (a) According to ZMUDA and ARMSTRONG (1974). The currents flowing both into and from the ionosphere are located in region A. B is the irregular region. (b) Magnetically quiet intervals according to IIJIMA and POTEMRA (1976b). (c) Magnetically disturbed period according to Iijima and Potemra. (d) Field-aligned currents in the polar cap according to SAFLEKOS et al. (1978).

The values of $j_{\|}^{M}$ shown in Figs. 3(a)-(d) involve a calibration of different effects including the magnetic field of the current induced in the Earth, the height of the current-carrying ionospheric layer, etc. The isolines contour the regions of equalintensity field-aligned currents. In Fig. 3(a) the field-aligned currents $j_{\|}^{M}\left(H_{0}\right)$ independent of the IMF direction cover the entire high-latitude region and are divided 


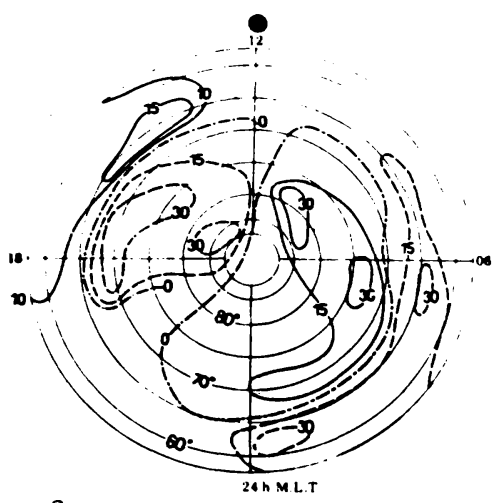

a

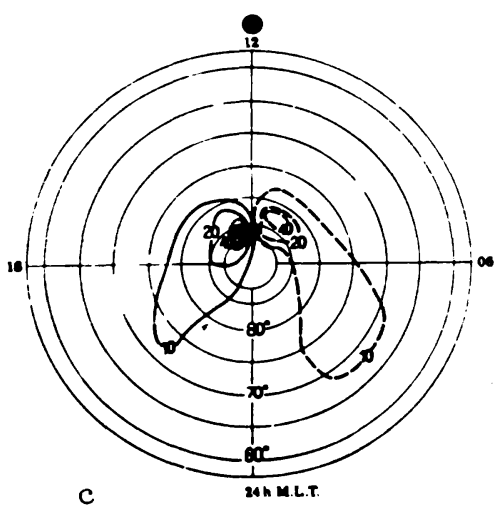

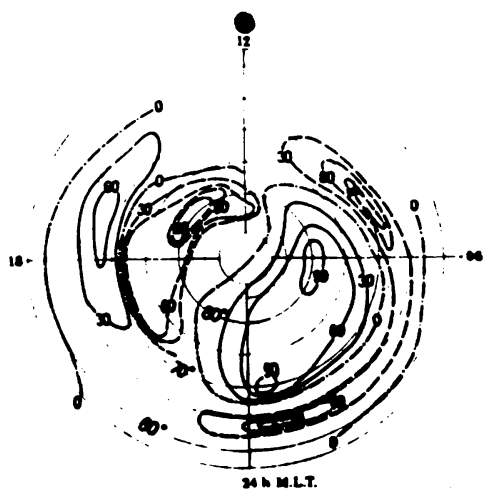

b

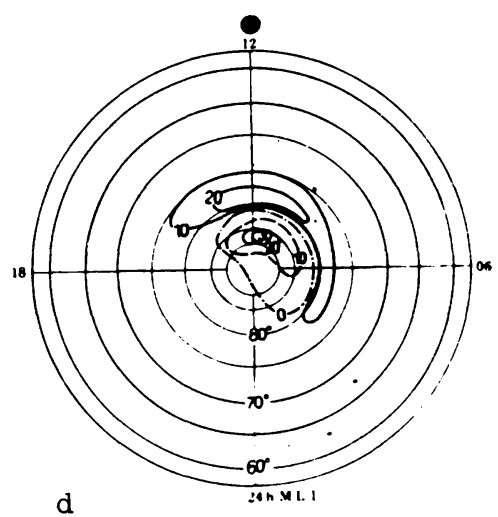

Fig. 3. The results of model-simulating for the space-time distribution of $j_{\|}$in summer (LEVITIN et al., 1977,1982 ) obtained in various interplanetary medium situations. The height-integrated ionospheric conductivity is taken to be homogeneous. The value of the isolines of $j_{\|}$are in units of $3 \times 10^{-9} \mathrm{~A} / \mathrm{m}^{2}$. Corrected geomagnetic latitude-local geomagnetic time coordinates are used. The solid lines show the flowing currents into the ionosphere and the dashed lines show the outflowing currents. (a) $\boldsymbol{B}_{\boldsymbol{y}}=\boldsymbol{B}_{z}=0$, $n=4$ particle $/ \mathrm{cm}^{3}, V=500 \mathrm{~km} / \mathrm{s}$. (b) $B_{z}=-1 \mathrm{nT}$. (c) $B_{z}=1 \mathrm{nT}$; (d) $B_{y}=1 \mathrm{nT}$.

by the 2200-1100 MLT meridian into two zones where the dawn (dusk) sector currents flow into (out of) the ionosphere in the poleward region and flow out of (into) the ionosphere in the equatorward region. The maximum density of the currents is $\sim 0.3 \times 10^{-6} \mathrm{~A} / \mathrm{m}^{2}$. The space-time structure of $j_{\|}^{M}\left(B_{z}<0\right)$ in Fig. 3(b) is similar to that of $j_{\|}^{M}\left(H_{0}\right)$. Figure 3(c) presents the distribution of $j_{\|}^{M}\left(B_{z}>0\right)$. The currents are located mainly in the polar cap region, flow into the ionosphere in the dusk sector, and flow out in the dawn sector. The currents $j_{\|}^{M}\left(B_{y}\right)$ controlled by the azimuthal IMF component are mainly concentrated near the dayside cusp and cover the day sector from dawn to dusk. For $B_{y}>0\left(B_{y}<0\right)$ the currents flow into (out of) the ionosphere on the equatorward side and flow out of (into) the ionosphere on the poleward side (Fig. 3(d)). The intensity and direction of $j_{\|}^{M}$ at a particular spatial point of the corrected geomagnetic latitude-local geomagnetic time coordinates is a function of the IMF. 
There is, therefore, a probability for a satellite to record $j_{\|}$different not only in value but also in direction at the same point, but at different UT time.

Like any model aimed at quantitative description of the regularities in the real situations, the model proposed must

(i) agree with the main regularities found experimentally in the field-aligned current distribution,

(ii) permit interpretation of individual observed deviations from such regularities,

(iii) predict new, yet unknown, regularities.

The $j_{\|}^{M}$ model (Figs. 3(a)-(d)) inferred from the observations of the geomagnetic field variations at the Earth surface satisfies the above conditions.

The general space-time $j_{\|}^{M}$ structure relevant to the IMF-independent geomagnetic variations (Fig. 3(a)) agrees with the structure of the large-scale field-aligned currents observed by TRIAD (Fig. 2(a)) although the $j_{\|}^{M}$ intensity in the maxima is somewhat lower due to the smearing of the field-aligned currents over a large area compared with the data of individual orbits. The permanent solar wind "streaming" around the magnetosphere cause the permanent existence of the region 1 and 2 field-aligned currents. Geomagnetic activity during $B_{z}<0$ periods is accompanied by an enhancement of the field-aligned currents in zones 1 and 2 with simultaneous conservation of their spatial distribution structure (Figs. 3(b) and 2(c)). The dependence of the zone $1 j_{\|}$intensity on the value of the southward $\left(B_{z}<0\right)$ component agrees with the results of the direct measurements (MCDIARMID et al., 1978; IIJIMA and POTEMRA, 1982). The zone 1 field-aligned current intensity increases as the $B_{z}<0$ component enhances, but does not vary with increasing $B_{z}>0$. This circumstance agrees with the proposed model because it may be interpreted as the existence of two field-aligned current sources in zone 1 of which one is independent of IMF, while the second is controlled by the southward IMF component. The $j{ }_{\|}^{M}$ distribution in the day sector is determined in a critical manner by the IMF intensity and direction. It is the IMF variability that is responsible for the occurrence of the irregular region in Fig. 2(a). By forming their various combinations, the currents $j_{\|}^{M}\left(H_{0}\right), j_{\|}^{M}\left(B_{z}\right)$, and $j_{\|}^{M}\left(B_{y}\right)$ give rise to the variability of the resultant field-aligned current in the high-latitude dayside sector. The distribution of $j_{\|}^{M}$ presented in Fig. 3(d) is in a good agreement with the model concept concerning the character of the $B_{y}$ component-controlled field-aligned currents proposed on other grounds by STERN (1973), LEONTYEV and LYATSKY (1974), VOLLAND (1975b), and LYATSKY (1978) and then inferred from the ISIS-2 data by MCDIARMID et al. (1979) and from TRIAD data by WILHJELM et al. (1978). The chaotic $j_{\|}$directions in zone 3 near local noon in Fig. 2(b) reflect the complicated distribution of the field-aligned currents and the difficulties faced in the analysis of the experimental data. The model calculations of the field-aligned currents in the polar cusp and polar cap (FELDSTEIN et al., 1982c) have shown that the $j \|$ distribution corresponds to the scheme of IIJIMA and POTEMRA (1976b) for certain interplanetary conditions and to the scheme MCDIARMID et al. (1979) for other conditions. It should be pointed out that the interpretation of the measurement data of particular satellite orbits in terms of one or another model is essentially dependent on the satellite trajectories.

FELDSTEIN et al. (1982a) discussed in detail the models for the large-scale magnetospheric field-aligned currents proposed by IIJIMA and POTEMRA (1976a, b), 
IIJIMA et al. (1978), and MCDIARMID et al. (1979) and concluded that the model shown in Fig. 3 explained the experimental data better.

It is now accepted that the high-latitude distribution of the field-aligned currents is largely controlled by IMF (POTEMRA et al., 1984). This is completely in agreement with the following conclusion (LEVITIN et al., 1977a): "The results of the satellite observations of the field-aligned currents have to be treated not on the basis of the calssification according to geomagnetic activity level, but as being contingent on the IMF orientation in the following basic situations: $B_{y}>0$ and $B_{z}>0, B_{y}>0$ and $B_{z}<0$, $B_{y}<0$ and $B_{z}>0, B_{y}<0$ and $B_{z}<0 "$.

Generally, the magnetic field variations detected by a satellite in the dawn sector are described by the two-layer model for the field-aligned currents flowing into the ionosphere in the near-pole part of the oval and flowing out in the equatorward part of the oval. However, the following cases of anomalous direction were reported by MCDIARMID et al. (1977) at 0400-1000 MLT; a field-aligned current flow out of the ionosphere at the poleward edge of the oval and into the ionosphere at its equatorward edge along the ordinary case within the oval. For all cases of the anomalous field-aligned currents the $B_{z}$ component was northward. However, the $j_{\|}$ direction was often normal at high values of $B_{z}>0$. These experimental data can be explained by the model displayed in Figs. 3(c) and (d) and were explained (LEVITIN $e t$ $a l ., 1977 \mathrm{~b}$ ) by the dependence of the field-aligned currents not only on $B_{z}$ but also on $B_{y}$ IMF component. In particular, on several orbit of the satellite the anomalous $j_{\|}$ direction was due to high values of the $B_{y}>0$ rather than to $B_{z}>0$. In the dawn sector, the normal $j_{\|}$direction $B_{z}>0$ must be observed for $B_{y}<0$. The field-aligned current model displayed in Figs. 3(a)-(d) has made it also possible to account for the occurrence of the three-layer $j_{\|}$system in the dawn sector on 1/3 of the S3-3 satellite orbits (MOZER et al., 1980).

In the proposed model, a field-aligned current system with maximum intensity at afternoon hours (current inflow) and prenoon hours (current outflow) appears in summer on the dayside of the polar cap during $B_{z}>0$ intervals. According to Fig. 3(c), the density of these currents reaches $\sim 10^{-7} \mathrm{~A} / \mathrm{m}^{2}$ at $B_{z}=+1 \mathrm{nT}$. The direction of the model field-aligned currents coincided with the zone 3 current direction (IIJIMA and POTEMRA, 1976b). However, the modeled currents cover the entire dayside polar cap sector up to the pole, and failed to form narrow bands at $\Phi<80^{\circ}$ (see Fig. 2(b)), and were controlled by the $B_{z}>0$ component, rather than by $B_{z}<0$ component. This bold prediction which was to be verified and could be used to test the applicability of all the methods for restoring the three-dimensional current system from the ground-based magnetic observation data. In 1977-1980 our $j_{\|}^{M}$ system for $B_{z}>0$ (Fig. 3(c)) provoked vivid discussions and certain distrust, while the method for separating the $B_{z}>0$ component-controlled magnetic field variations on the Earth's surface was cast doubted by BAZARZHAPOV et al. (1979), and PUDOVKIN and ZAITSEVA (1983).

The existence of the $B_{z}>0$ component-controlled field-aligned currents in the dayside sector of polar cap which flow into the ionosphere in the afternoon sector and flow out in the prenoon sector was convincingly demonstrated by MCDIARMID et al. (1980) and by SAFLEKOS and POTEMRA (1980). The preliminary analysis of the TRIAD data (POTEMRA et al., 1980), demonstrated intense $j_{\|}$in the polar cap for $B_{z}=9.8 \mathrm{nT}$. COWLEY $(1981 \mathrm{~b})$ found a fairly high correlation between the intensity of the transverse disturbances of magnetic field inferred from the TRIAD and ISIS-2 
data and positive values of the $B_{z}$ component. The existence of a large scale fieldaligned current system in polar cap during summer which is controlled by the $B_{z}>0$ component was also confirmed recently by IIJIMA (1984) and ARAKI et al. (1984). By now, the most comprehensive study of the space-time distribution of field-aligned currents during intensive $B_{z}>0$ condition has been carried out by IIJIMA et al. (1984). Figure 4(a) presents the composite diagram of the spatial distribution of the largescale field-aligned currents inferred from the data of nine MAGSAT orbits traversing the high latitudes of the southern hemisphere on January 8, 1980. From 1100 to 2300 UT, $B_{z}$ was stable and equal to $\sim 8 \mathrm{nT}$. Therefore, the consecutive orbits may be treated as a scanning of the steady-state spatial distribution of the field-aligned currents over the polar cap in summer. The large-scale field-aligned currents flowing into the ionosphere in the prenoon sector and out in the afternoon sector can be seen on the polar cap dayside on $\Phi>80^{\circ}$. The field-aligned currents at $\Phi<80^{\circ}$ belong to zones 1 and 2. Figure 4(b) presents the intensities of magnetic disturbances along the orbits. The near-pole currents are much more intensive than in zones 1 and 2 and reach $\sim 0.8 \times 10^{-6} \mathrm{~A} / \mathrm{m}^{2}$ in the day sector at $\Phi>83^{\circ}$. These estimates have been inferred from the satellite magnetograms on the assumption that the currents $j_{\|}$are of the form of infinite "curtains" extended across the orbits. Since in reality the $j_{\|}$ configuration may be treated as "curtain" type in only a rough approximation, the real $j_{\|}$may differ from these estimates (KISABETH and ROSTOKER, 1977; DREMUHINA et al., 1985). The comparison of Figs. 4(a) and (b) with Fig. 3(c) shows that the positions, directions, and intensities of the currents are similar. The data of the MAGSAT and TRIAD measurements presented by IIJIMA et al. (1984) must be treated as excellent evidence for the existence of the $B_{z}>0$ component-controlled large-scale field-aligned current system in the near-pole regions. The characteristic features of the system, namely, the location of the most intensive currents on the dayside, flowing into ionosphere in the afternoon sector and out in the prenoon sector, the relationship to the $B_{z}>0$ component intensity, were infrerred from the observations of the magnetic field variations on the Earth's surface. The results of IIJIMA et al. (1984) confirm the existence of this $B_{z}>0$ current system rather than actually discovering a "new Birkeland current system".

The field-aligned current model displayed in Figs. 3(a)-(d) is identical to the pattern of the field-aligned currents in the dayside cusp region for the northern hemisphere for various IMF directions proposed by TROSHICHEV and GIZLER (1980) and Troshichev (1984). In contrast to the model which gives the field-aligned current direction and intensity for each hour of MLT and for each degree of latitude, the pattern gives but a general idea about the location of the currents by substituting a line for the regions where they are present. A somewhat different pattern of fieldaligned current distribution was proposed by PUDOVKIN et al. (1984). The main differences are due to the location and direction of the $j_{\|}$controlled by the $B_{y}$ component. Namely, the field-aligned currents $j_{\|}$are located exclusively in zone 1, their directions are different at prenoon and afternoon hours and do not depend on the $B_{y}$ sign of the $B_{y}$ component. Present-day satellite data unambiguously confirm the pattern presented here as reported by TROSHICHEV (1984), WILHJELM et al. (1978), DOYLE et al. (1981), VENNERSTROM et al. (1984). 

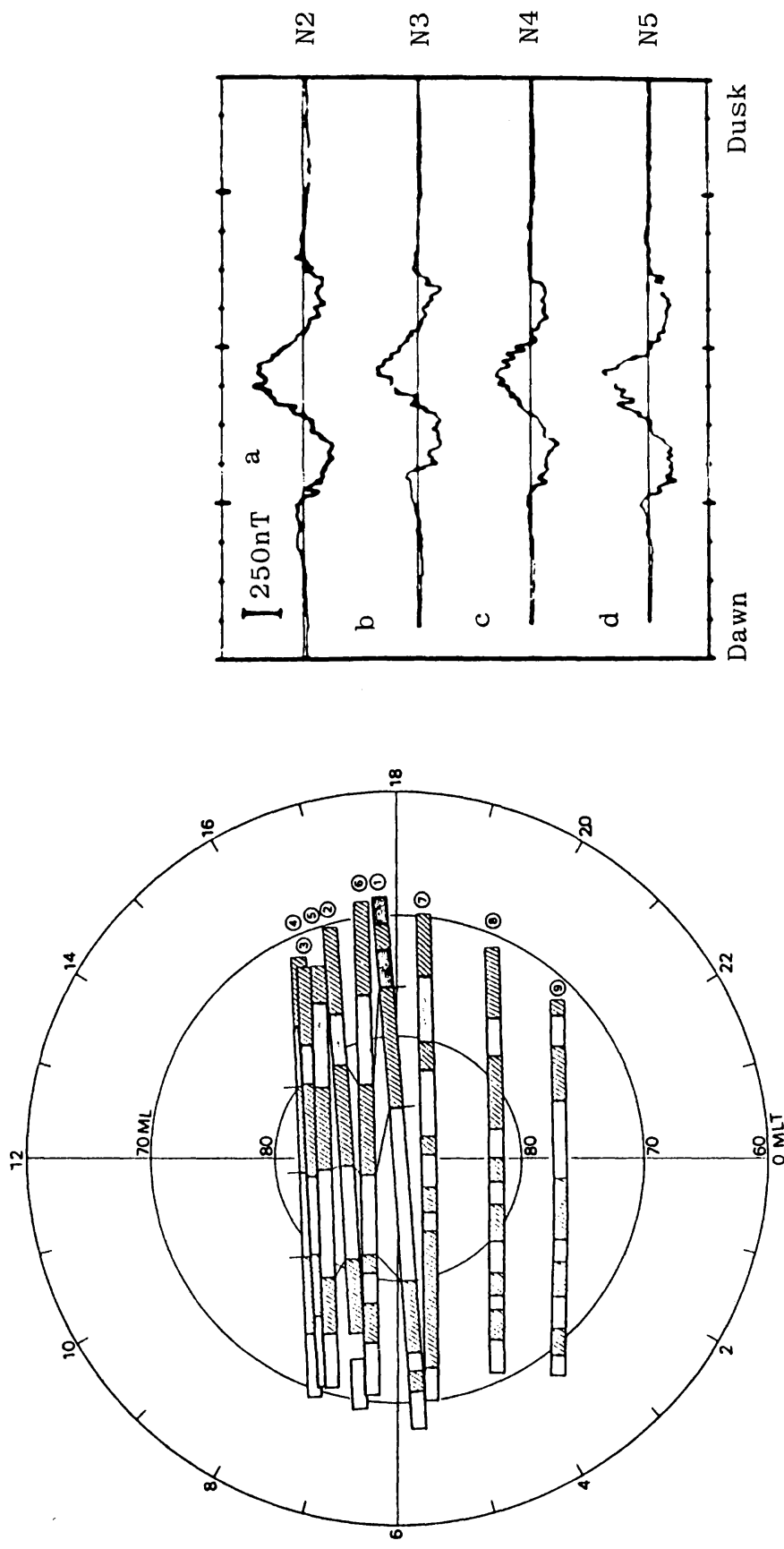

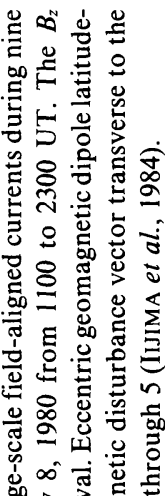

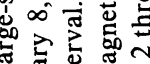

莺壳芯.

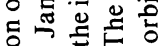
돌

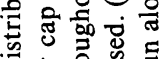

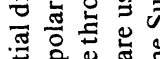
若 过

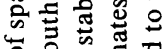
요욜

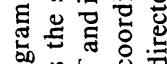

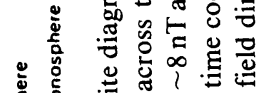

范

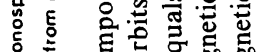
응 क

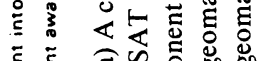

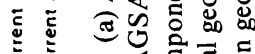
ปे ○迹 
5. Determination of the Electromagnetic Parameters of the Upper Atmosphere in Terms of a Model for Nonuniform Ionospheric Conductivity

In the case of nonuniform ionospheric conductivity, the electric field potential is determined from the second-order partial differential equation

$$
-\frac{1}{\tau}\left[\left(\tau \Sigma_{\mathrm{H}} \phi_{\theta}\right)_{\Theta}+\frac{1}{\tau}\left(\Sigma_{\mathrm{H}} \phi_{\Lambda}\right)_{\Lambda}\right]+\frac{1}{\tau}\left[\left(\Sigma_{\mathrm{P}} \phi_{\Lambda}\right)_{\Theta}-\left(\Sigma_{\mathrm{P}} \phi_{\Theta}\right)_{\Lambda}\right]=\frac{1}{\tau}\left(\tau \Psi_{\theta}\right)_{\Theta}+\frac{1}{\tau^{2}} \Psi_{\Lambda \Lambda}
$$

where $\Theta$ and $\Lambda$ are respectively the colatitude and longitude in spherical coordinates; $\tau=\sin \Theta$. In (4) $\Psi$ is the current function related unambiguously to the variation of the horizontal magnetic field on the Earth's surface and calculated for various classes of the geomagnetic field variations. In the numerical calculations of $\phi$ on the basis of (4), the height-integrated ionospheric conductivity tensor is usually specified on a grid with a $1^{\circ}$ step in latitude and a $15^{\circ}$ step in longitude ( $1^{\mathrm{h}}$ of local geomagnetic time). The total integral Hall and Pedersen conductivities are composed of the conductivities resulting from both ionization by solar radiation and particle precipitation. The solar UV input produces height-integrated conductivities that vary as the cosine of the solar zenith angle (METHA, 1979; ROBINSON and VONDRAK, 1984). This conductivity source represents a background upon which the contribution from auroral charged particles should be added (WALLIS and BUDZINSKI, 1981; SPIRO et al., 1982). The models of high-latitude height-integrated ionospheric conductivities proposed elsewhere were discussed and compared in (REIFF, 1984; FELDSTEIN and LEVITIN, 1984). Different conductance models are used as input to solve the equation (4) numerically.

KAMIDE and RICHMOND (1982) calculated electric fields and ionospheric and field-aligned currents on the basis of ground-based magnetic records used with drastically different models for ionospheric conductivity. In has been shown that the ionospheric current patterns are in practice independent of, the field-aligned current patterns are weakly dependent on, and the electric field patterns depend strongly on the choice of the conductivity. Our calculations have proved to be in complete agreement with these conclusions. They also substantiate the use of the uniform ionospheric conductivity model to calculate the summer pattern of the field-aligned currents from the ground-based magnetic variation data in the pioneer studies of such kind.

The application of more realistic conductivity models will little change the $j_{\|}$ distribution shown in Fig. 3. The uniform ionospheric conductivity approximation seems to be sufficient in summer to calculate the estimates of the ionospheric and field-aligned currents from the ground-based data. The experimental data of magnetic and electric fields at low altitudes do not contradict this conclusion (Wilhjelm et al., 1978; Primdahl and SPANGSLEV, 1977; PRIMDAhl et al., 1979). The analysis of the combined measurements of magnetic and electric fields from the low-altitude satellites suggests that the summer ionospheric conductivity may be treated as nearly uniform. In the following sections we describe the results of model calculations of the electrostatic potential and the electric fields from the magnetic field variations on the Earth's surface and the inhomogeneous ionospheric conductivity models. As a rule, for the corpuscular part we use the WALLIS and BUDZINSKI (1981) 
model. These calculations fail to introduce essential corrections in the results for all of summer and part of equinox seasons. In winter, when solar UV induced conductivity is practically absent, the situation changes. The necessity arises to have more accurate information on the high-latitude distribution of the corpuscular conductivity which is extremely variable and may differ noticeably in the particular cases from the mean model distributions.

6. The Electrostatic Potential and Convection in the High-Latitude Ionosphere as a Function of Interplanetary Conditions

6.1 The main features of the spatial distribution of $\phi^{\mathrm{M}}(\Phi, M L T)$ in different seasons Numerical model calculations (AFONINA et al., 1983; FELDSTEIN et al., 1984a, b; LEVITIN et al., 1984), of the potential $\phi^{\mathrm{M}}$ at the observation point $(\Phi$, MLT) can be represented by

$$
\phi^{\mathrm{M}}\left(\Phi, \operatorname{MLT}, B_{y}, B_{z}\right)=\phi_{Y}^{\mathrm{M}}(\Phi, \operatorname{MLT}) B_{y}+\phi_{Z}^{\mathrm{M}}(\Phi, \operatorname{MLT}) B_{z}+\phi_{0}^{\mathrm{M}}(\Phi, \operatorname{MLT})
$$

where $B_{y}$ and $B_{z}$ are the hourly means of the IMF vector components at the moment when $\phi$ is determined. The model contains different sets of the coefficients $\phi_{Y}^{\mathrm{M}}, \phi_{Z}^{\mathrm{M}}, \phi_{0}^{\mathrm{M}}$ relevant to the calculations for the $B_{z} \geqslant 0$ component (use is made of the WALLIS and BUDZINSKI (1981) model for the height-integrated ionospheric conductivity at $K_{p}<3$ ) and at the $B_{z}<0$ component (use is made of the height-integrated conductivity model at $\left.K_{p}>3\right)$. The distributions of $\left.\phi_{Y}^{\mathrm{M}}(\Phi, \mathrm{MLT}), \phi_{Z}^{\mathrm{M}}, \mathrm{MLT}\right)$, and $\phi_{0}^{\mathrm{M}}(\Phi, \mathrm{MLT})$ show how the electric field in the high-latitude ionosphere responds to the IMF components $B_{y}= \pm 1 \mathrm{nT}, B_{z}= \pm 1 \mathrm{nT}$, and $B_{z}=B_{y}=0$, respectively. As a result we can obtaine the distribution of $\phi^{\mathrm{M}}\left(\Phi, \mathrm{MLT}, B_{y}, B_{z}\right)$ under a particular IMF situations.

Figure 5 shows $\phi_{Y}^{\mathrm{M}}, \phi_{Z}^{\mathrm{M}}$ and $\phi_{0}^{\mathrm{M}}$ in the $\Phi$-MLT coordinate. The isolines present the equal values of the electrostatic potential in $\mathrm{kV}$. Each panel shows the IMF conditions situations, the season, the universal time for calculating the distribution of the height-integrated ionospheric conductivity due to solar UV radiation from the Sun ( $\mathrm{UT}_{\mathrm{m}}$ corresponds to the coincidence between the geomagnetic and geographic axes), the $K_{p}$-index characterizing the high-latitude ionospheric conductivity due to the corpuscular precipitations in terms of the WALLIS-BUDZINSKI (1981) model.

These "elementary" potential distributions are characterized by the following features. Two convective vortices exist in summer when $B_{z}=B_{y}=0$, i.e., in case of quasiviscous interaction of the solar wind plasma with the magnetosphere (the $\phi^{\mathrm{M}}$ contour lines represent convective flow paths of magnetospheric plasma with $\phi^{\mathrm{M}}>0$ characterizing the counterclockwise convection). The centers of the vortices are located at $\Phi \sim 77^{\circ}$ for $0300-0400$ MLT (the dawn vortex) and at $\Phi \sim 80^{\circ}$ for $1600-1700 \mathrm{MLT}$ (the dusk vortex). The potential is $12.6 \mathrm{kV}$ at the dawn vortex center and $16.8 \mathrm{kV}$ at the dusk vortex center. In its spatial dimensions the dusk vortex exceeds the dawn vortex.

The $B_{y}$ component produces a single convective vortex centered about the corrected geomagnetic pole. The convection direction in the vortex depends on the sign of the IMF azimuthal component namely for $B_{y}>0\left(B_{y}<0\right)$ the convection in the northern hemisphere is clockwise (counterclockwise). The inverse relationship between the $B_{y}$ and convection directions is observed in the southern hemisphere. 

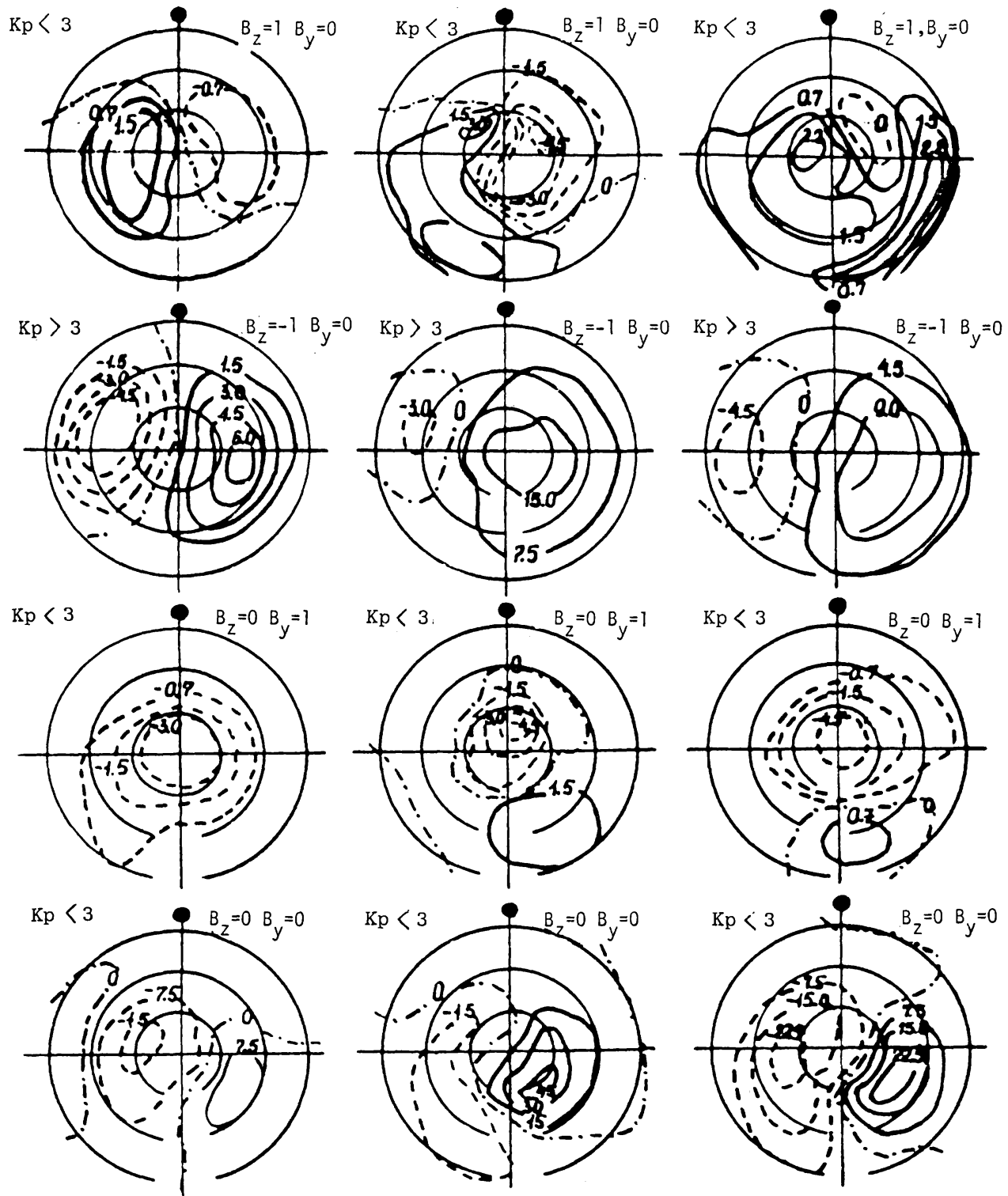

Fig. 5. The isolines of the electrostatic potential $\phi^{\mathrm{M}}$ in $\mathrm{kV}$ during three seasons in various interplanetary medium conditions. The geographic axis coincides with the geomagnetic axis $\left(\mathrm{UT}_{m}\right)$. The corrected geomagnetic latitude $80^{\circ}, 70^{\circ}$ and $60^{\circ}$-local geomagnetic time coordinates are used (LEVITIN et al., 1984).

Like the quasiviscous interaction, the southward IMF vector component produces two convective vortices of the same direction. The $B_{z}<0$ condition enhancements both dawn and dusk vortices which are permanently present in the 
high-latitude ionosphere due to the continuous interaction of the solar wind with the magnetosphere.

In summer, the center of the dawn vortex produced by the $B_{z}=-1 \mathrm{nT}$ component is located at $\Phi \sim 75^{\circ}$ for $\sim 0500 \mathrm{MLT}$; where $\phi^{\mathrm{M}} \sim 7.5 \mathrm{kV}$. The dusk vortex center is located at $\Phi \sim 75^{\circ}$ for $\sim 1700 \mathrm{MLT}$; where $\phi^{\mathrm{M}} \sim-6 \mathrm{kV}$. In this case the spatial dimensions of the dawn vortex exceed those of the dusk vortex.

The northward IMF vector component produces two convective vortices with the convection directions opposite to those in the vortices produced by the viscous interaction and the $B_{z}<0$ component. In their dimensions, these vortices are much smaller and are located in the dayside polar cap sector. In summer the dawn vortex center is located at $\Phi \sim 82^{\circ}$ for $\sim 0900$ MLT (for where $\phi^{\mathrm{M}} \sim-1.5 \mathrm{kV}$ ), and the dusk vortex center at $\Phi \sim 82^{\circ}$ for $\sim 1500 \operatorname{MLT}\left(\phi^{\mathrm{M}} \sim 1.8 \mathrm{kV}\right)$.

In winter, the vortices are rearranged. At $B_{y}=B_{z}=0$, the dawn vortex center is located at $\Phi \sim 80^{\circ}$ for $\sim 0200-0300$ MLT (where $\phi^{\mathrm{M}} \sim 54 \mathrm{kV}$ ), and the dusk vortex center is at $\Phi \sim 72^{\circ}$ for $\sim 2000 \operatorname{MLT}\left(\phi^{\mathrm{M}} \sim-21 \mathrm{kV}\right)$. The dawn vortex produced by the $B_{z}<0$ component is spatially larger than of the dusk vortex and covers almost the entire high-latitude region (the maximum value of $\phi^{\mathrm{M}}$ is $\sim 21 \mathrm{kV}$ ). The $B_{y}$ componentproduced main vortex gets narrow, and an additional vortex appears at night hours. At the equinoxes, the distributions of $\phi_{Y}^{\mathrm{M}}, \phi_{Z}^{\mathrm{M}}$ and $\phi_{0}^{\mathrm{M}}$ are intermediate between summer and winter.

The representation of the ionospheric convection pattern by the sum of elementary cells coincide with the three types of magnetospheric convection cells suggested by BURCH et al. (1985), and REIFF and BURCH (1985), namely, the merging cells ( $B_{z}<0$ in our case, see Fig. 5(b)), the viscous cells (at $B_{z}=B_{y}=0$ in our case, see Fig. 5(d)) and the lobe cells (controlled by $B_{y}$ in our case, see Fig. 5(c), and by $B_{z}>0$, see Fig. 5(a)). In Fig. 5, however, the ionospheric manifestation of the viscous cells cover also exist in the polar cap, while the lobe cells are extended to the auroral zone. Therefore, a more significant overlapping of the magnetospheric cells may occur. As a result, the ionospheric systems are somewhat different than the magnetospheric systems predicted by BURCH et al. (1985) and REIFF and BURCH (1985). However, the coexistence of three types of convection cells as elements of single convection model (BURCH et al., 1985) is completely in agreement with our model.

Under the real conditions, a particular $\phi^{\mathrm{M}}(\Phi, \mathrm{MLT})$ pattern depends on the IMF orientation and may be much more complicated. To illustrate the total effect of the $B_{y}$ and $B_{z}$ components on the potential distribution pattern. Figure 6 shows the summer distributions of $\phi^{\mathrm{M}}(\Phi, \mathrm{MLT})$ for $B_{z}=0$ and $B_{y}= \pm 6 \mathrm{nT}, B_{y}=0$ and $B_{z}= \pm 4 \mathrm{nT}, B_{z}=-4$ $\mathrm{nT}$ and $B_{y}= \pm 6 \mathrm{nT}$.

The model distributions of $\phi_{Y}^{\mathrm{M}}, \phi_{Z}^{\mathrm{M}}$ and $\phi_{0}^{\mathrm{M}}$ make it possible to estimate the potential difference, $\Delta \phi$, across the polar cap. In the situation with $B_{y}=\mathrm{B}_{z}=0$ (the case of quasiviscous interaction), $\Delta \phi_{0}^{\mathrm{M}} \simeq 29 \mathrm{kV}$ but varies somewhat depending on the conductivity model applied. According to AFONINA et al. (1979) and SERGEEV and KUZNETSOV (1981), the value of $\Delta \phi_{0}$ is proportional to the square of the solar wind velocity. When the southward IMF increases by $1 \mathrm{nT}$ the value of $\Delta \phi^{\mathrm{M}}$ is $13.5 \mathrm{kV}$, and when the northward IMF increases by $1 \mathrm{nT}$ the potential difference is $3.3 \mathrm{kV}$ and is directed inversely from dusk to dawn and appears in the polar cap day sector. TROSHICHEV (1984) obtained approximately the same value of $\Delta \phi^{\mathrm{M}}$ on the assumption of uniform height-integrated ionospheric conductivity in summer with 


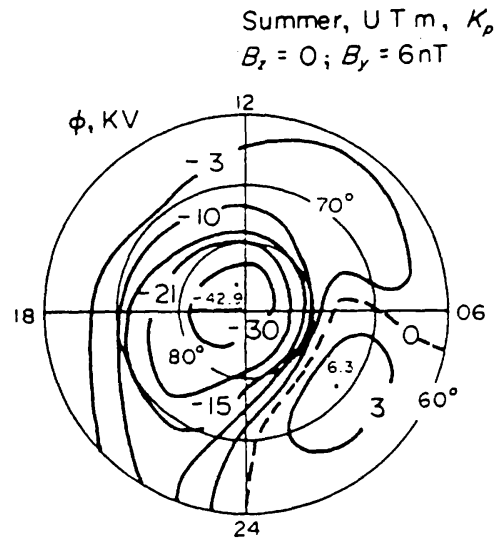

(a)

Summer, $U T \mathrm{~m}, K_{p}<3$ $B_{z}=4 n T ; B_{y}=O n T$

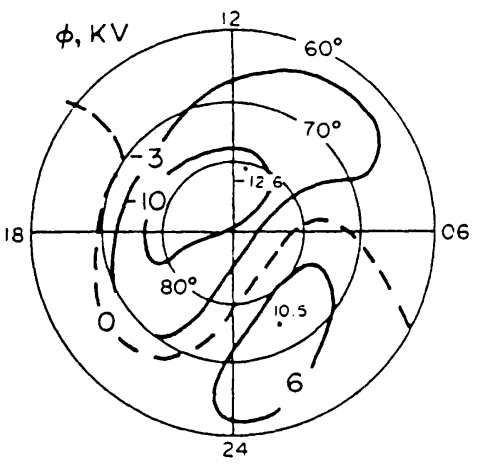

(c)

Summer, $\cup \top \mathrm{m}, K_{p}>3$ $B_{z}=-4 n T ; B_{y}=6 n T$

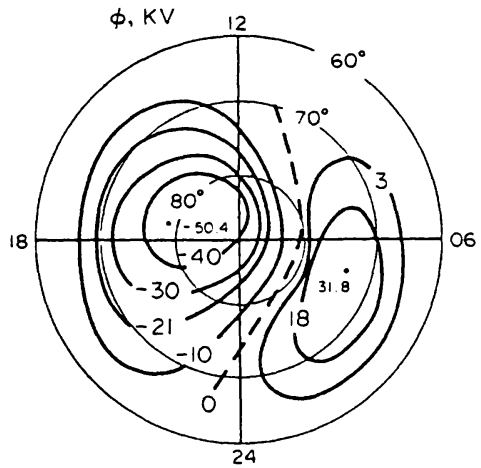

(e)
Summer, U T $m, K_{p}<3$

$B_{z}=0 ; B_{y}=-6 n T$

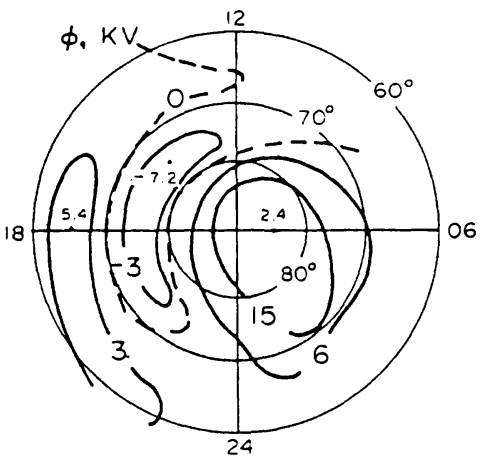

(b)

Summer, $\cup T m, K_{D}>3$ $B_{z}=-4 n T ; B_{y}=O n T$

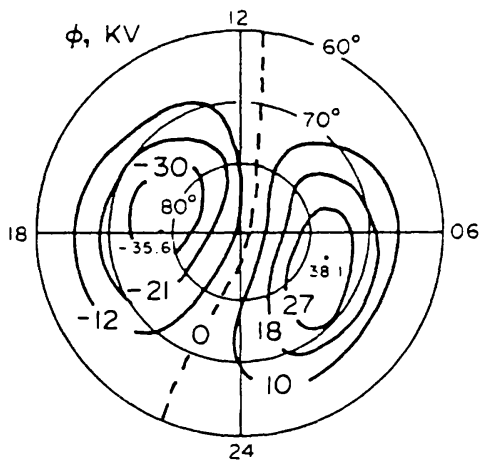

(d)

Summer, UT m, $K_{p}>3$

$B_{z}=-4 n T ; B_{y}=-6 n T$

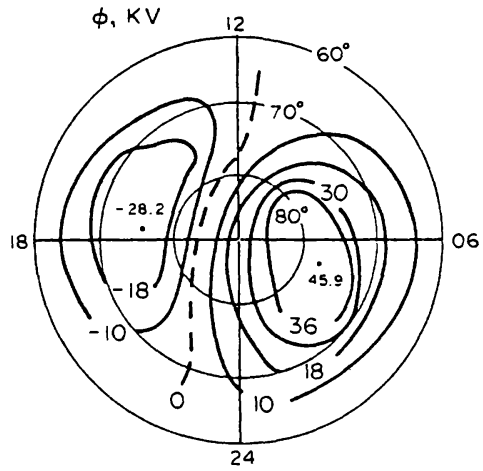

(f)

Fig. 6. The isolines of the electrostatic potential $\phi^{\mathrm{M}}$ in $\mathrm{kV}$ in summer for various interplanetary conditions (FELDSTEIN et al., 1984a). 
$\Sigma_{\mathrm{H}}=10$. The model value of $\Delta \phi_{0}^{\mathrm{M}} \approx 29 \mathrm{kV}$ in the $B_{z}=B_{y}=0$ situation agrees with the results of the direct measurements over the polar cap from the low-orbiting satellites AE-C and D (REIFF et al., 1981), S3-2 (DOYLE and BURKE, 1983), and S3-3 (WYGANT et al., 1983). They report a $\sim 30 \mathrm{kV}$ IMF-independent potential difference over the polar cap. However, this value is much larger than the $\Delta \phi$ value measured by MOZER (1984) in the magnetospheric boundary region which was $\sim 5 \mathrm{kV}$ in case of viscous interaction. Further satellite and radar measurements to be taken to establish the ultimate relation between the electric field induced by viscous interaction and merging of the IMF and the magnetospheric magnetic field on the magnetopause and the value of $\Delta \phi$ across the polar cap.

LyATSKy (1978), Crooker (1979), CoWley (1982, 1984), and TroshicheV (1984) have analyzed the IMF effect on the high-latitude convection. The $B_{z}$ component controls the merging at the magnetopause giving rise to a decrease or increase of the potential difference across the polar cap. The $B_{y}$ component gives rise to an increase of the convection intensity in the dawn (dusk) sector of the northern hemisphere when $B_{y}>0\left(B_{y}<0\right)$. This feature of the convection behaviour was first noted by HEPPNER (1972) and confirmed afterward by numerous studies including JøRGENSEN (1981) and ClAUER et al. (1984). GALPERIN et al. (1978) have demonstrated the dependence of the convection direction in the dayside cusp on the $B_{y}$ component sign. The possible effects of the IMF components on the magnetospheric convection were discussed by STERN (1983), COWLEY (1981a, 1982), and AleXeEv and BelenkAYA (1983a, b) using a model for the superposition of a homogeneous IMF on the dipolar geomagnetic field. Despite its simplicity, the models account for numerous fine scale features of the convective motion distribution in the magnetosphere. It should be noted that the convection model at $B_{z}>0$ presented in Fig. 6 differs from the analogous schemes proposed by CROOKER (1979), TroshicheV (1984), and others.

The model calculations have shown that the concept of narrow ( \pm 1 hour near noon) throat proposed in (HEELIS, 1984 and the references therein) for the convection pattern on the polar cap day side fails to be reflected in the $\phi^{\mathrm{M}}(\Phi$, MLT) distributions inferred from the ground-based magnetic observations.

The transition from sunward convection in the dawn and dusk sectors to the antisunward pattern in the polar cap takes the form of a rotational reversal. The change from the sunward velocity direction to antisunward in Fig. 6 is due to the rotation of the convection velocity vector with a moderate increase in velocity. These results agree with the radar experiments (EVANS et al., 1980; FOSTER et al., 1981; JØRGENSEN et al., 1984) which show that the convection in the polar cap occurs over a broad time interval from 0800 to 1600 MLT. The measurements (FOSTER et al., 1985) have shown that plasma enters the polar latitudes across a rotational convection reversal which span at least 3 hours of local time in the noon sector. Something like a throat, but much more extended than \pm 1 hour near noon, appears at high values of $B_{y}$ and $B_{z}<0$ (FELDSTEIN et al., 1984a).

\subsection{Intercomparison of the $\phi^{\mathrm{M}}$ patterns inferred from the ground-based magnetic field variations}

It is presently possible to compare two independent calculations of $\phi^{\mathrm{M}}$ using the magnetogram-inversion schemes. FELDSTEIN et al. (1984a) used hourly data from the 
standard high-latitude observatories for 1968, the FAERMARK (1980) algorithm, and the MeTha (1979) and WALlis-BudZINSKI (1981) models for height-integrated ionospheric conductivity. FRIIS-CHRISTENSEN et al. (1985) used the 20-min data from the chain of magnetometers along the west coast of Greenland for the summer seasons of 1972 and 1973, the KAMIDE et al. (1981) algorithm, and the heightintegrated ionospheric conductivity models similar to those used by KAMIDE and MATSUSHITA (1979). In both cases, the magnetic field variations on the Earth's surface controlled by the $B_{z}$ and $B_{y}$ components and independent of IMF were separated using the same method.

Figure 7 presents the results of FRIIS-CHRISTENSEN et al. (1985) for the various IMF orientations. The $\phi\left(\Phi\right.$, MLT) patterns for $B_{z}=B_{y}=0$ is shown in the center. Eight other patterns for various combinations of $\boldsymbol{B}_{z}$ and $\boldsymbol{B}_{y}$ are displayed in the $\boldsymbol{B}_{\boldsymbol{y}}-\boldsymbol{B}_{z}$ coordinate system, with $\sqrt{B_{z}^{2}+B_{y}^{2}}=5 \mathrm{nT}$. Although the calculations were made using different algorithms and different input data, the general features of the potential distribution proved with several exception turnings to be in a good agreement with FELDSTEIN et al. (1984a) shown in Fig. 6.

The two-cell convection pattern has been obtained for the $B_{z}=B_{y}=0$ condition with the zero potential isoline near the geomagnetic pole and a $\sim 30-35 \mathrm{kV}$ potential difference across the polar cap. The $B_{z}>0, B_{y}=0$ condition is characterized by a decrease of the dawn vortex, by a shift of its focus near to midnight, and by a clockwise rotation of the distribution as a whole. The two-cell convection pattern is preserved, although the electric field direction may change from dawn-dusk to dusk-dawn in a limited latitude region of the day sector. In Fig. 7 it is seen from the isolines character in the day sector at $\Phi \sim 85^{\circ}$, and in Fig. 6 isoline surge is absent due to the spatial smoothing. However, the two studies do not confirm the occurrence of the four vortex convection system in high latitudes during the $B_{z}>0$ intervals as proposed by BURKE et al. (1979). The $B_{y}>0\left(B_{y}<0\right), B_{z}=0$ situations are accompanied by an enhanced convection in the dawn (dusk) sectors.

KAMIDE et al. (1983) and MISHIN et al. (1984) calculated the high-latitude potential distributions using their magnetogram inversion techniques. The magnetic field variations at 107 magnetic observatories during the CDAW-6 period for March 22, 1979 were used as input data. WOLF and SPIRO (1984) have found a reasonably good agreement between the results of the two studies by comparing visually between the potential distribution plots at 10.30 UT, 11.10 UT and 11.40 UT on March 22, 1979. Figure 8 is a quantitative comparison between the distributions of $\phi^{\mathrm{M}}$ along the meridional cross-section seperated by three hours of MLT. The respective KAMIDE et al. (1983) meridional cross-sections were averaged for the 10 minutes which fell within the 30-45 minutes intervals used by MISHIN et al. (1984). Noticeable discrepancies exist during all the substorm phases in the character of the latitude variations of $\phi^{\mathrm{M}}$ along fixed meridian. Since the values of magnetic field variations used as input data were identical, the discrepancies must be unexpected. They cannot be ascribed to differences in the conductivity models used in calculations. The allowance for the difference in the boundary conditions also fails to significantly improve the agreement between the results of the two groups of researchers. It is desirable to find the reasons for the disagreement, which is necessary for further perfection of the method of potential restoration from the ground-based magnetograms. BAKER and KAMIDE (1985) also revealed some discrepancies for the CDAW-6 period at the comparison of 


\section{ELECTRIC POTENTIAL}
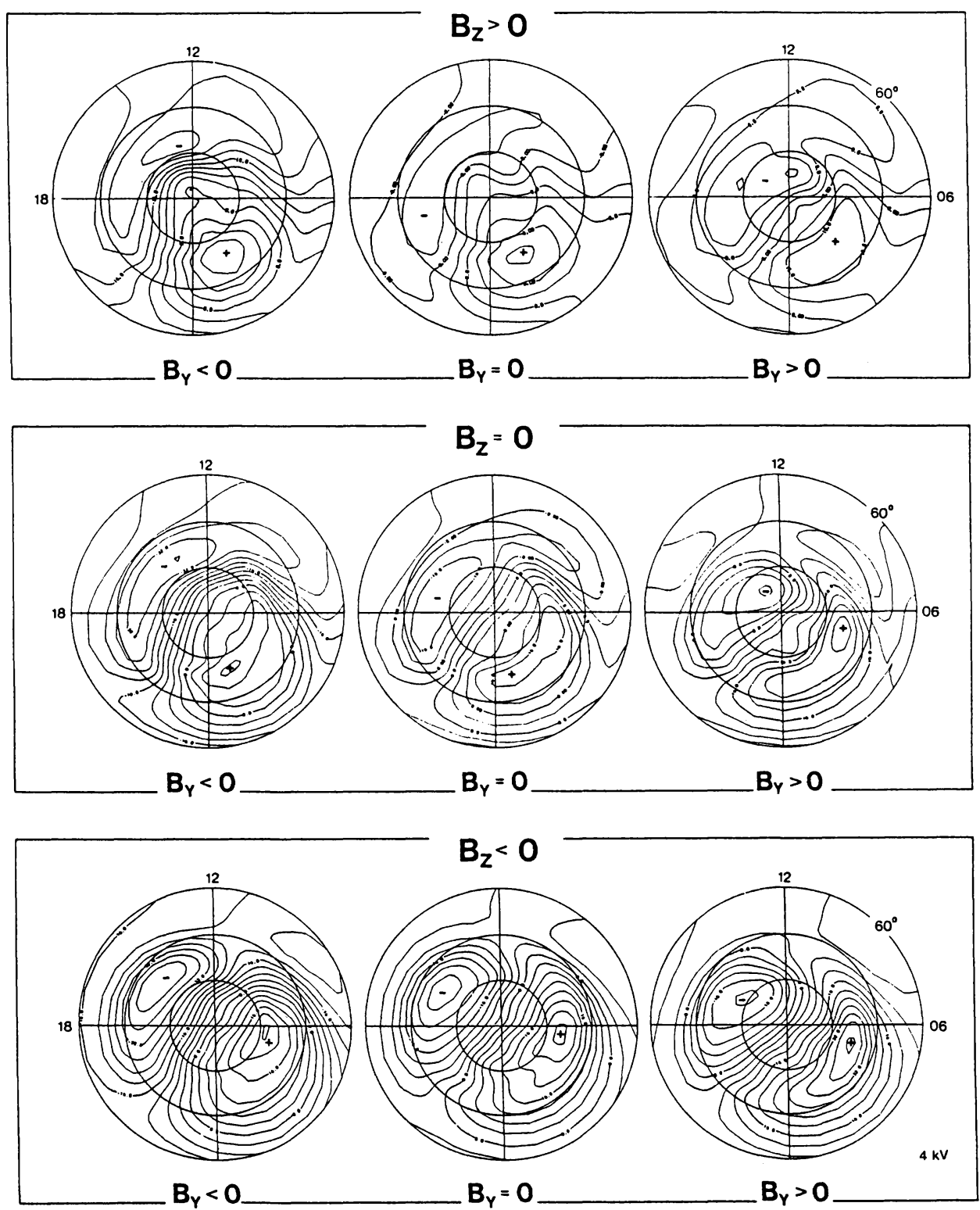

Fig. 7. The electrostatic potential distribution for various IMF conditions from FRIIS-CHRISTENSEN $e t$ al. (1985). The isolines are spaced $4 \mathrm{kV}$ apart. The $0 \mathrm{kV}$ isoline runs through the pole.

radar electric fields with the KRM output.

The electric field model obtained by statistical processing of the incoherent radar data (FosTER, 1983) was scaled to the model for the potential $\phi(\Phi$, MLT) at northern-hemisphere auroral latitudes (FOSTER, 1984). Figure 9 shows the potential 


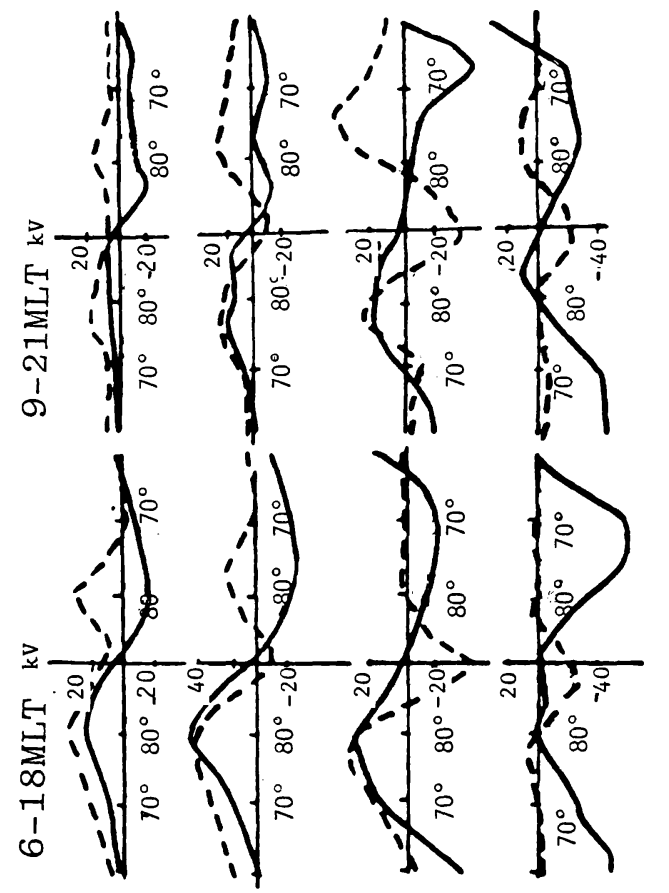

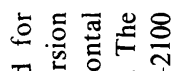

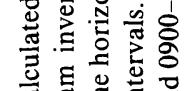

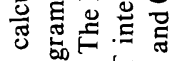
త] 过 혐 응

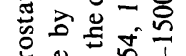
造 。

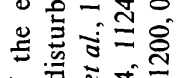

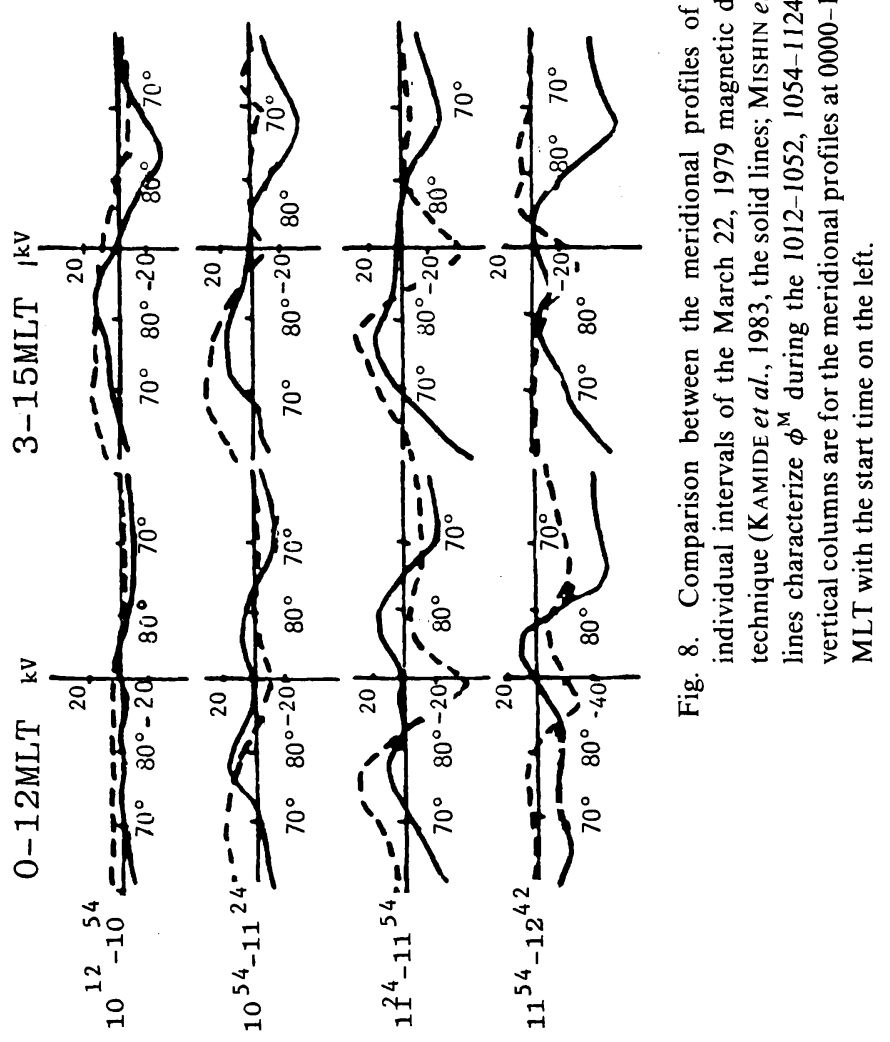




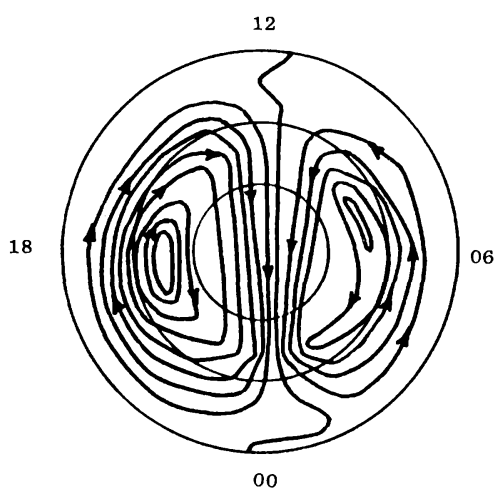

00

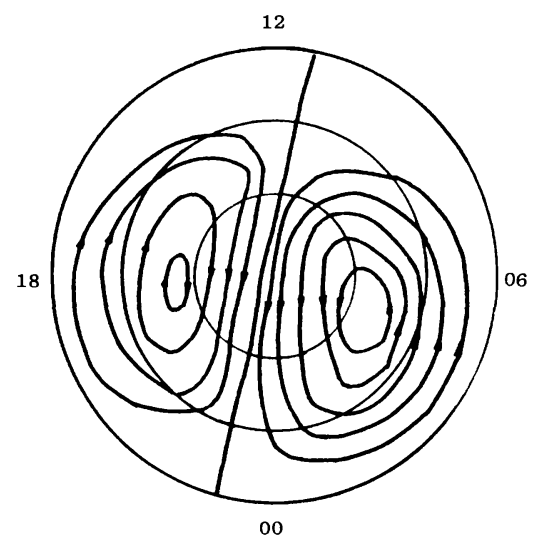

$\Delta \phi=5 \mathrm{kV}$

Fig. 9. The space-time distribution of the electrostatic potential in summer in terms of the FOSTER (1984) model (on the left) and the LEvitin et al. (1984) model (on the right) in the $\boldsymbol{B}_{z}=-2 \mathrm{nT}, \boldsymbol{B}_{y}=-3 \mathrm{nT}$ situation. The isolines are spaced $5 \mathrm{kV}$ apart.

isolines in this model (at the left) and in the model LEVITIN et al. (1984) (at the right) for the IMF situation with $B_{z}=-2 \mathrm{nT}, B_{y}=-3 \mathrm{nT}$. The results of comparing between the models indicate that the average potential distribution restored from the geomagnetic data reflects the global features of the electric field potential $\phi(\Phi, M L T)$ inferred from direct measurements.

HeElis (1984), Potemra et al. (1984), and ReifF and BuRCH (1985) have proposed the models of high-latitude plasma convection dependent on the $B_{y}$ component. HEELIS (1984) has analyzed the convection distribution along the AE-C orbits and constructed a detailed model for the convection on the day side of the polar cap for $B_{z}<0$ and various $B_{y}$ directions. Figure 10 shows the vectors of the convection velocity $V_{d}$ along the satellite trajectories (to the left) and the model $V_{d}\left(\Phi\right.$, MLT, $B_{y}$, $B_{z}$ ) distributions calculated in terms of the model LEVITIN et al. (1984). The model describes adequately the large-scale features of the experimental data, in particular the changes of the convection directions in the respective IMF situations. It may be expected, therefore, that the model is close to reality throughout the high-latitude region. Figure 11 shows the model convection distributions (HEELIS, 1984) for the dayside hemisphere at $\Phi \geq 60^{\circ}$ to the left and the convection velocity vector distributions in terms of the electric field model (LEVITIN et al., 1984) for the same region (at the right). The values $B_{z}<0$ and $B_{y} \gtrless 0, B_{y} \gtrless \gtrless 0$ adopted by Heelis correspond to $B_{z}=-2 \mathrm{nT}, B_{y}= \pm 3 \mathrm{nT}, B_{y}= \pm 10 \mathrm{nT}$ in the LEVITIN et al. (1984) model. In the $B_{y}=+10 \mathrm{nT}$ situation, plasma flows to the near-pole region in the dawn sector at an increased velocity at $\Phi \sim 80^{\circ}$ HeELIS et al. (1976) called the throat. In the $B_{y}=-10 \mathrm{nT}$ IMF situation, the encounter of the sunward convective streams occurs near noon at $65^{\circ}<\Phi<75^{\circ}$ and the plasma flows into the near-pole region at the highest velocity through the dusk sector. Thus, the plasma flows into the near pole region mainly through the dawn (dusk) sector at $B_{y}>0\left(B_{y}<0\right)$. However, the flux change over from sunward to antisunward direction is different in the HEELIS (1984) and LEVITIN et al. (1984) models. Perhaps the local convective whirlings seen in Fig. 


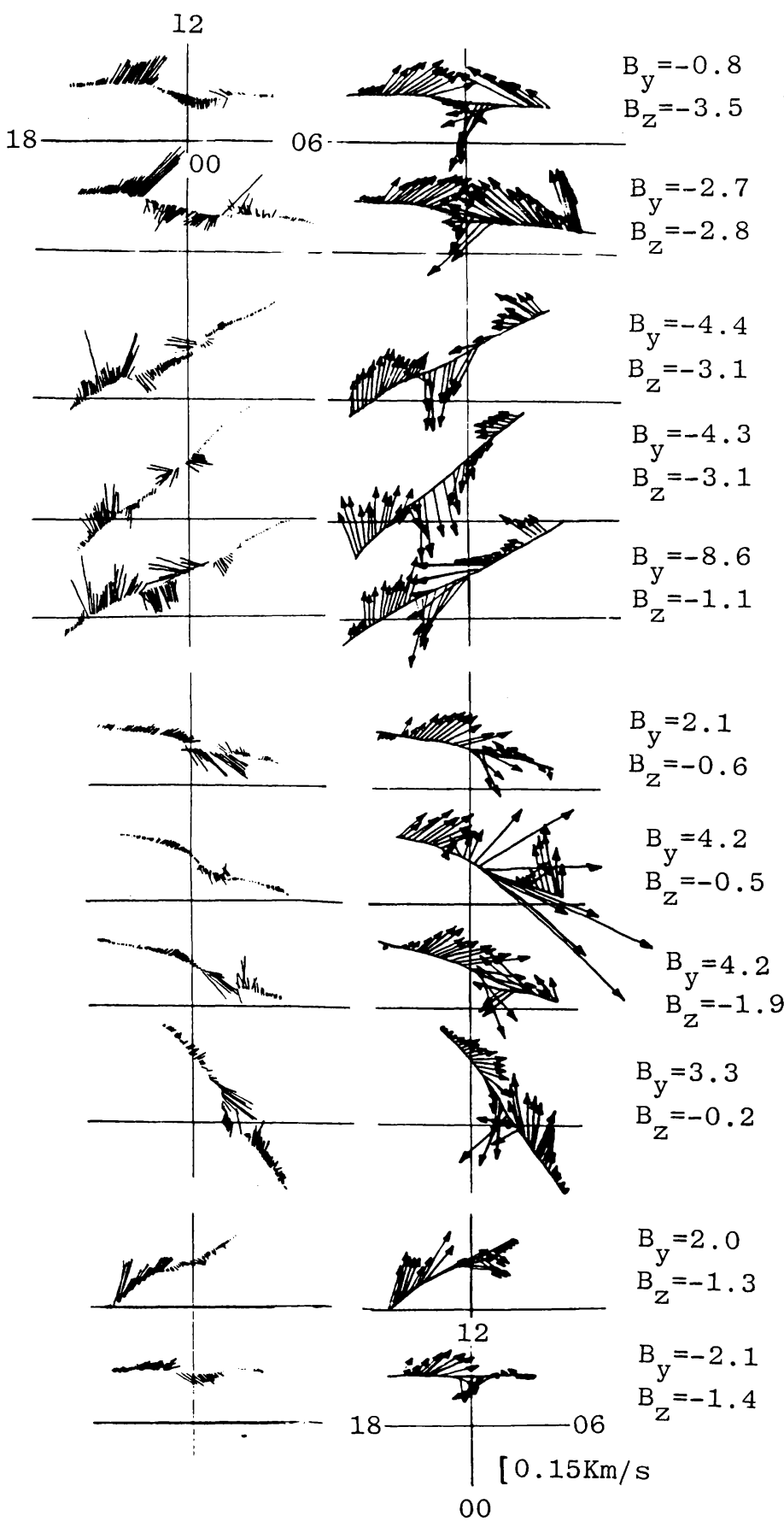

Fig. 10. The distribution of the convection velocity $V_{d}$ along the AE-C orbits. The experimental data (HEELIS, 1984) are shown on the left and the model data (LEVITIN et al., 1984) on the right for the IMF situations indicated. 


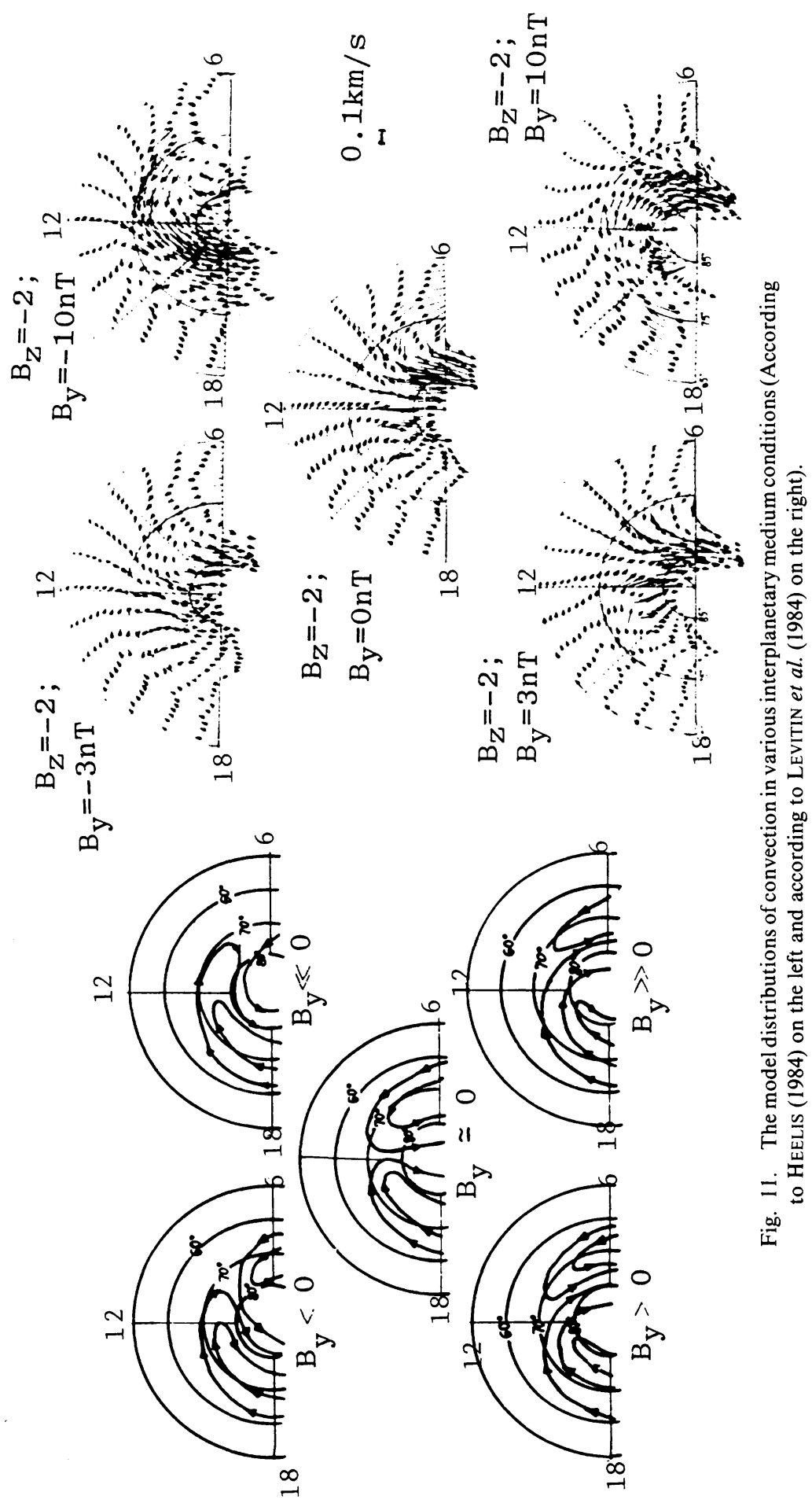


11 at the right in higher latitudes may be interpreted in terms of the throat, namely at $B_{y}=10 \mathrm{nT}, 0600-0800 \mathrm{MLT}$ on $\Phi \sim 75-78^{\circ}$ and at $B_{y}=-10 \mathrm{nT}, 0600-0800 \mathrm{MLT}$ at $\Phi \sim 75-83^{\circ}$.

The presence of the inverse (sunward) convection in the polar cap during the $\boldsymbol{B}_{z}>0$ intervals, which was noted first in the geomagnetic data (MAEZAWA, 1976; KUZNETSOV and TROSHICHEV, 1977; and many other works) and then in the satellite data (BURKE et al., 1979), has motivated researchers to set forth the hypothesis that four convective vortices exist in the high-latitude ionosphere during such intervals (BURKE et al., 1979; REZHENOV et al., 1980; REZHENOV, 1981; AKASOFU and ROEDERER, 1983; TROSHICHEV, 1984; REIFF and BURCH, 1985 and the references therein).

The model (LEVITIN et al., 1984) predicts a complicated spatial pattern of convection depending on the IMF which is considerably different from such idealized configuration probably because the satellite observations yield true information on the convection distribution only along satellite orbits. The extrapolation to the entire high-latitude region depends in a certain manner on the researcher's intuition. Therefore, such extrapolation should be treated with caution.

Figure 12 shows the isolines of the potential $\phi(\Phi, M L T)$ for IMF conditions with $B_{z}=6,8,15 \mathrm{nT}$ and $B_{y}=0$ in accordance with the LEVITIN et al. (1984) model. These distributions demonstrate that even for large positive values of $B_{z}$ the region with the dusk-dawn electric field direction covers but a limited sector of the dayside polar cap and does not extend to the night side. An interesting fact should be noted, namely the clockwise potential rotation of the potential pattern with increasing $B_{z}$ while the conductivity remains constant. The convection pattern which is symmetric with respect to the noon-midnight merdian at $B_{z}<0$ turns to the dawn-dusk meridian for $B_{z} \approx 8 \mathrm{nT}$. The same rotation was described by YASUHARA et al. (1983) relevant to the conductivity variations in the auroral oval.

The effect of $B_{y}$ IMF on high-latitude convection during northward IMF was discussed in POTEMRA et al. (1984), REIFF and BURCH (1985) and the references therein. Figure 13 shows the appropriate schematic convection diagrams according to Potemra et al. (the upper row), Reiff and Burch (the middle row) and Levitin et al. (the lower row) for the $B_{z}=10 \mathrm{nT}, B_{y}=0, \pm 10 \mathrm{nT}$ situations. Significant differences in the proposed convection models can be seen. We expect that the recent satellite and radar observations will make it clear to what extent the proposed models are able to simulate the real situation.

7. The Dependence of the Electric Field in the High-Latitude Ionosphere on Interplanetary Parameters

Numerical model calculations (AFONINA et al., 1983; LEVITIN et al., 1984) of the electric field $E$ (the meridional component is positive directed towards the corrected geomagnetic pole, the azimuthal component is positive directed towards the magnetic east) at the observation point $(\Phi$, MLT) can be represented by

$$
E\left(\Phi, \operatorname{MLT}, B_{y}, B_{z}\right)=E_{y}(\Phi, \operatorname{MLT}) B_{y}+E_{z}(\Phi, \operatorname{MLT}) B_{z}+E_{0}(\Phi, \operatorname{MLT})
$$

and is determined by the structure of the potential $\phi\left(\Phi\right.$, MLT, $\left.B_{y}, B_{z}\right)$ which is 


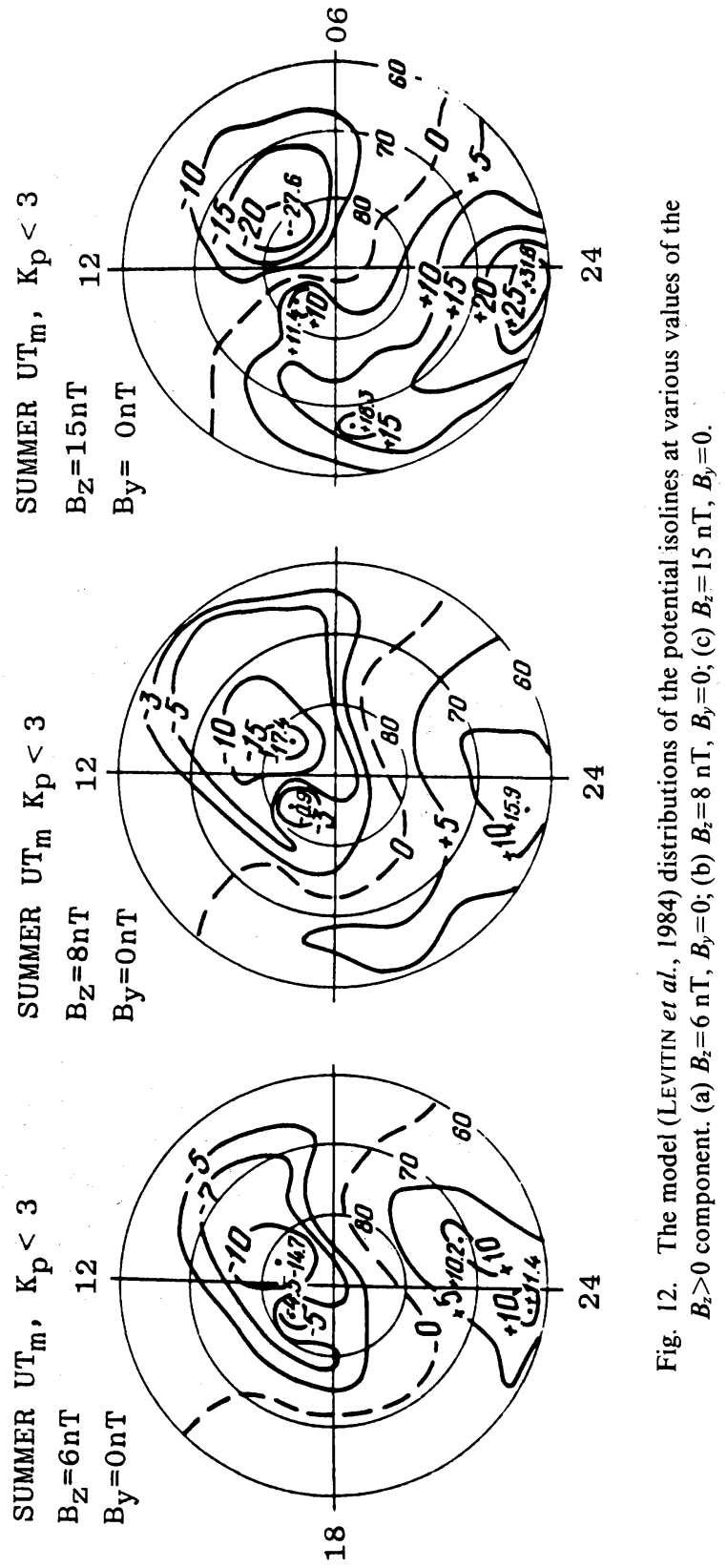




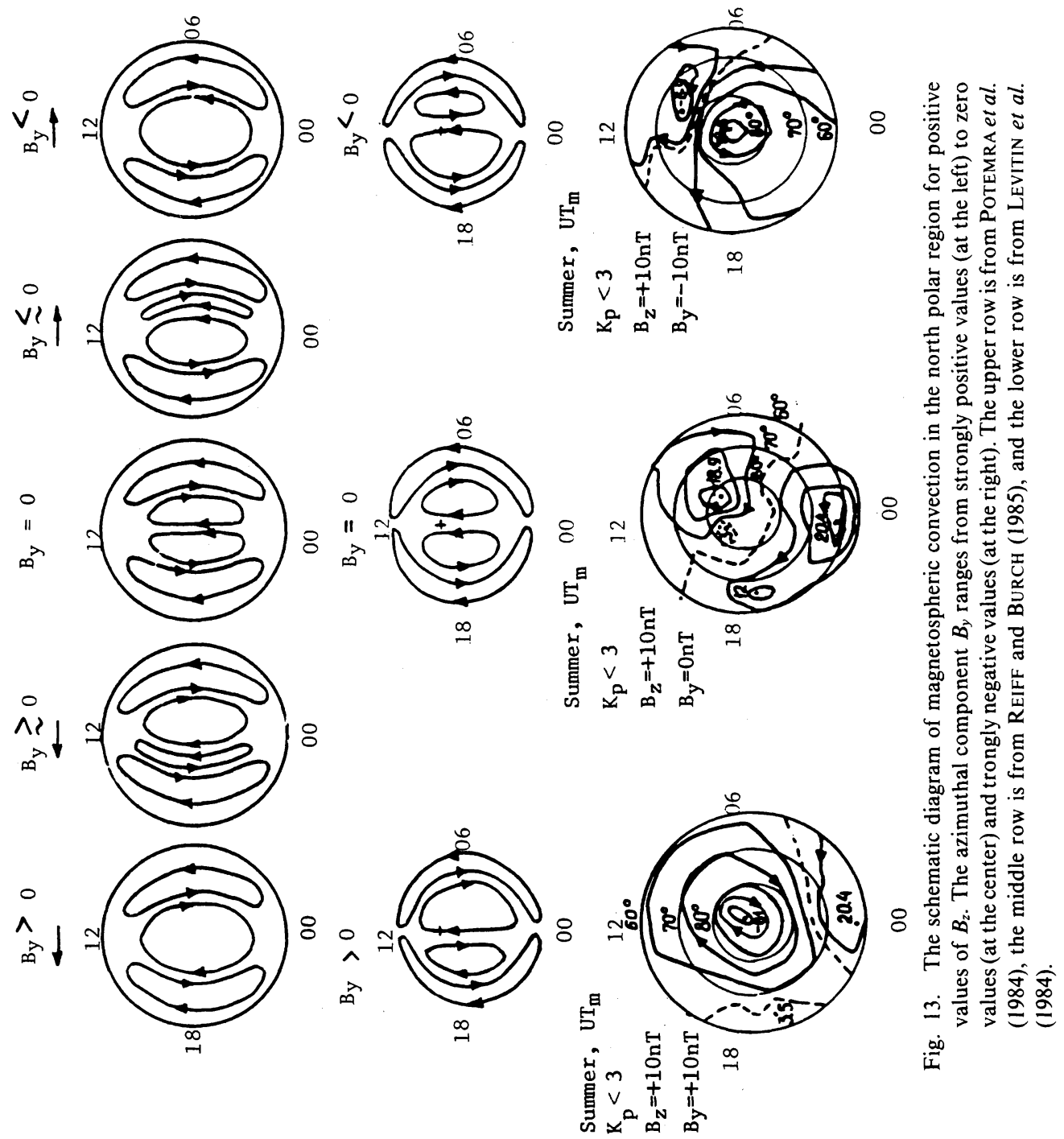


associated with $\boldsymbol{E}$ by the relation $\boldsymbol{E}=-\nabla \phi$.

The distributions of $\boldsymbol{E}_{y}, \boldsymbol{E}_{z}$ and $\boldsymbol{E}_{0}$ are determined by the respective distributions of $\phi_{y}^{\mathrm{M}}, \phi_{z}^{\mathrm{M}}$ and $\phi_{0}^{\mathrm{M}}$ and reflect the response of the electric field in the high-latitude ionosphere to the variations of $B_{y}=1 \mathrm{nT}, B_{z}= \pm 1 \mathrm{nT}$, and $B_{z}=B_{y}=0$.

The model distributions of $\boldsymbol{E}_{y}, \boldsymbol{E}_{z}, \boldsymbol{E}_{0}$ predict a complicated pattern of the spatial structure of the total electric field which can only describe the general electric field distribution due to the assumptions adopted (for example, VollaND 1975a; KAWASAKI, 1975; STERN, 1977; IVANOV and EVDOKIMOVA, 1975).

This circumstance should be borne in mind when analyzing the satellite data. The comparison between the results obtained on close, but not identical, orbits through the $\Phi>60^{\circ}$ region should be made very cautiously. Sufficiently pronounced spatial variations of the electric field must also be taken into account when interpreting the complex experiments, like those of HEPPNER et al. (1971) when the electromagnetic measurements are taken simultaneously but the Earth observations point spaced several degrees of latitude apart (FELDSTEIN et al., 1984a).

\subsection{Comparison of the model calculations of $\boldsymbol{E}(\Phi, M L T)$ with the HEPPNER (1972) observations on the dawn-dusk meridian}

The OGO-6 observations were used in HEPPNER $(1972 ; 1977)$ to obtaine the typical configurations (labelled A, B, C, D, E, F, G, H, I, K, SC, RC) of the meridional electric field component in the northern high-latitude region in summer along the dawn-dusk meridian. Figure 14 presents the latitudinal profiles of the meridional component $E_{m}$ on the dawn-dusk meridian in summer (a) and winter (b). In all the profiles, the positive values of $E_{m}$ correspond to the dawn-to-dusk field. Each figure shows the $E_{m z}(\Phi)$ distributions for $B_{z}>0$ and $B_{z}<0$ (above), $E_{m 0}(\Phi)$ for $B_{z}=B_{y}=0$ (in the middle), and $E_{m y}(\Phi)$ for $B_{y}=1 \mathrm{nT}, B_{z}<0$ and $B_{y}=-1 \mathrm{nT}, B_{z}>0$. In the LEVITIN $e t$ al. (1984) model, $E_{m y}\left(B_{y}<0\right)=-E_{m y}\left(B_{y}>0\right)$ and, therefore, two plots at the bottom of the figure are sufficient for all the possible situations with $B_{z} \gtrless 0, B_{y} \gtrless 0$ to be described. In the $B_{z}=B_{y}=0 \mathrm{IMF}$ condition, as follows from Fig. 14, the polar cap electric field is directed from dawn to dusk. In the auroral zone, the field sign reverses. The permanent existence of the dawn to dusk field and its relatively high intensity compared with the rest of the terms in Eq. (6) provide for the predominant dawn-todusk direction of $E_{m}$ in the polar cap. However, this electric field is inhomogeneous (is stronger in the dawn sector). Correspondingly, Heppner's configuration B must be observed not only at $B_{z}=B_{y}=0$, but also in case of low $B_{z}$ and $B_{y}$ values.

The electric field $E_{m z}\left(B_{z}<0\right)$ configuration is of the Heppner type A. When that field is added to the field $E_{m 0}$ the field $E_{m z}\left(B_{z}<0\right)$ gives rise to an increased field intensity in the polar cap and in the auroral zone. The electric field controlled by the northward IMF vector component $E_{m z}\left(B_{z}>0\right)$ composed with $E_{m 0}$ will, on the contrary, give rise to decreased field intensity in the polar cap and in the auroral zone. This latter condition may yield the Heppner type I configuration with high-intensity $B_{z}>0$ fields.

The electric field $E_{m y}\left(B_{y}>0\right)$ gives rise to a decreased field $E_{m 0}$ in the dusk sector and to an increased $E_{m 0}$ in the dawn sector. The direction of the field $E_{m y}\left(B_{y}>0\right)$ is the same as the $E_{m z}\left(B_{z}>0\right)$ direction in the dusk sector on $\Phi>84^{\circ}$ and is opposite in the dawn sector on $\Phi<87^{\circ}$. The $E_{m y}$ distributions at $B_{z}>0$ and $B_{z}<0$ differ little from each other. Thus, the model distributions shown in Fig. 14 may account for all the 

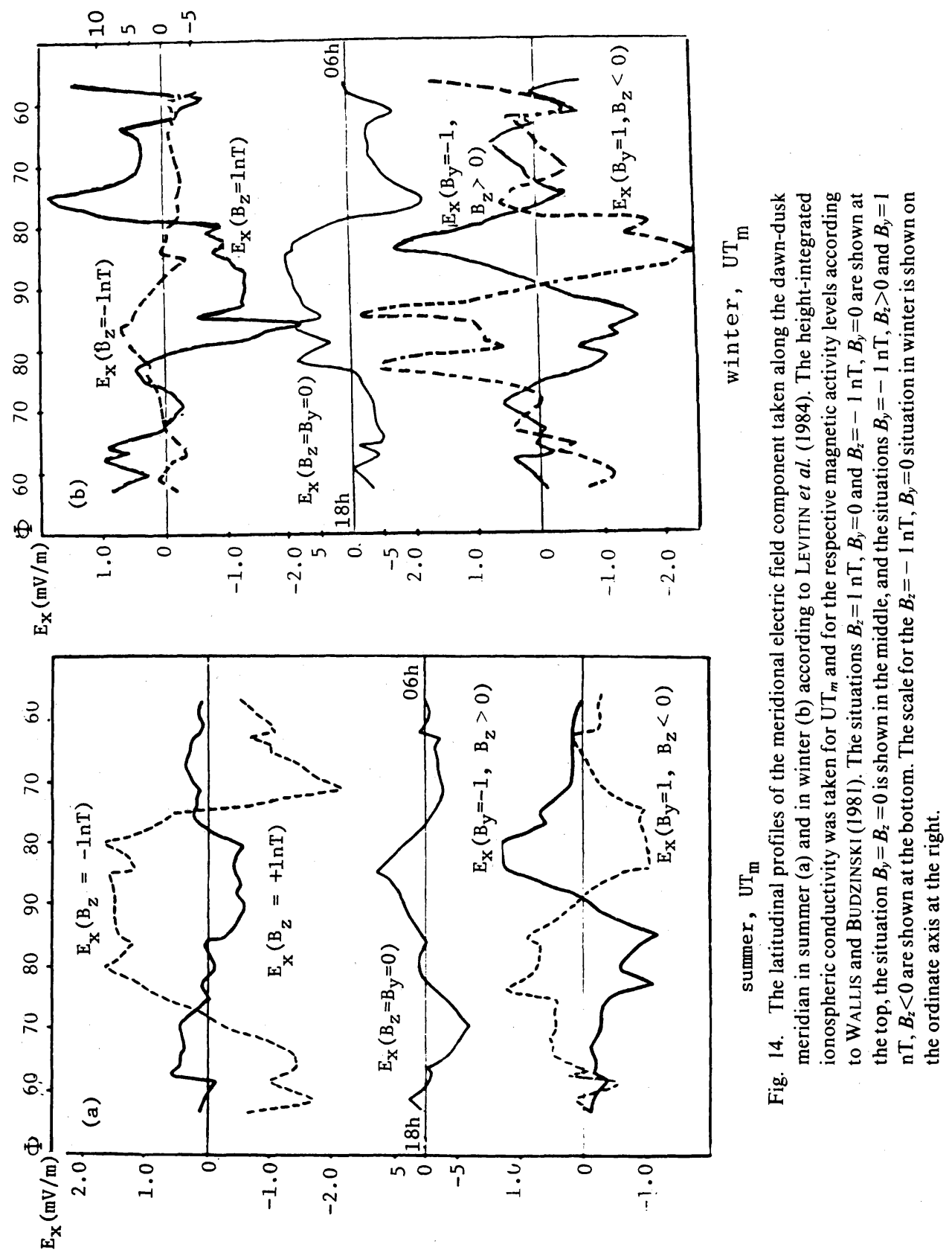
configurations described by HEPPNER (1972) when analyzing the OGO-6 data.

The model distributions of $E_{m}(\Phi)$ in winter (Fig. 14(b)) not only preserve the main features of the summer distributions but also exhibit their own charcteristic features. The field $E_{m z}\left(B_{z}<0\right)$ in the polar cap (the scale for this configuration is shown in Fig. 14(b) along the ordinate axis to the right) is much different from the Heppner type A structure. The field controlled by the $B_{z}<0$ is inhomogeneous in the polar cap and is directed mainly from dawn to dusk in the dusk sector where it takes its maximum values. The configuration of the electric field $E_{m 0}$ in polar cap in winter is closer to the Heppner configuration A than in summer.

Thus, it follows from the proposed model for $E(\Phi$, MLT), that the polar cap electric field along the dawn-dusk meridian is highly inhomogeneous and depends strongly on the IMF orientation. The model predicts a decrease of the polar cap electric field with values of positive $B_{z}$. According to the model, the potential difference along the dawn-dusk meridian through the polar cap cannot be treated as an unambiguous index of the intensity of the processes in the Earth's magnetosphere.

First, the highest potential difference through the polar cap is not located in all cases on the 0600-1800 MLT meridian (in certain IMF conditions such difference may be observed, for example, on the 1200-2400 MLT meridian, see Fig. 13). Second, even the zero potential difference does not indicate that the solar wind-magnetosphere interaction has ceased. In terms of the proposed model, the potential difference existing permanently in the magnetosphere is superposed on an additional field of opposite direction. As a result, the potential difference decreases. It should be borne in mind that the $B_{y}$ component-controlled current system is produced by the polar cap electric field whose potential difference across the polar cap is almost zero. By no means, however, does this fact imply that the processes of the electric field generation in the magnetosphere have ceased.

\section{Conclusions}

Due to the efforts of recent years the quantitative concept of interplanetary medium connection with the electric and magnetic fields, field-aligned and ionospheric currents in the Earth's magnetosphere has been created. Ground observations combined with radar, rocket and polar satellite data together with numerical methods of solving the equation for the electrostatic potential have made it possible to find the large-scale features of the electromagnetic weather in the Earth's environment resulting from the changes in the interplanetary medium. Some results of such efforts are described above. They show the importance of ground-based observations of the geomagnetic field variations for the purposes of continuous detection of the changing conditions in the space. Conversely, the conditions in the space make it possible to appraise the electromagnetic conditions in the Earth's magnetosphere. The development of the proposed methods will be an extremely important advance toward quantitative understanding of large-scale magnetosphere-ionosphere coupling. The improvement of the network of ground-based magnetic stations, especially in the polar cap, more exact and detailed data about ionospheric conductivity will permit the local features of the electromagnetic state of the magnetosphere and their detailed structure to be studied further. 


\section{REFERENCES}

Afonina, R. G., B. A. Belov, A. E. Levitin, and Y. I. Feldstein, About the geomagnetic field variations controlled by the velocity and density of the solar wind, in Interplanetary medium and magnetospheric events, edited by K. G. Ivanov, pp. 54-60, IZMIRAN, Moscow, 1979.

Afonina, R. G., B. A. Belov, A. E. Levitin, M. Yu. Markova, Y. I. Feldstein, and M. V. Fiskina, Connection of components of the interplanetary magnetic field with field-aligned currents in high latitudes of the northern hemisphere, Geomag. and Aeron., 20, 1073-1083, 1980.

Afonina, R. G., B. A. Belov, V. Yu. Gaidukov, L. A. Dremuhina, A. E. Levitin, D. S. Faermark, Y. I. Feldstein, Yu. Z. Demidova, and M. Yu. Markova, The model of the electric field potential in high-latitude ionosphere, in Geomagnetic Variations, Electric Fields and Currents, edited by A. E. Levitin, pp. 25-28, IZMIRAN, Moscow, 1983.

AKasofu, S-I., Polar and Magnetospheric Substorms, 273 pp., D. Reidel, Dordrecht, 1968.

AKASOFu, S.-I., Physics of Magnetospheric Substorms, 587 pp., D. Reidel, Dordrecht, 1977.

AKASOFU, S.-I., Energy coupling between the solar wind and the magnetosphere, Space Sci. Rev., 28, $121-190,1981$.

AKASOFU, S.-I., The magnetospheric currents: An introduction, in Magnetospheric currents, edited by T. A. Potemra, pp. 29-48, AGU, Washington, 1984.

AKasofu, S.-I. and C.-I. MENG, A study of polar magnetic substorms, J. Geophys. Res., 74, 293-313, 1969.

AKasofu, S.-I. and S. Chapman, Solar-Terrestrial Physics, 879 pp., Clarendon Press, Oxford, 1972.

Akasofu, S.-I. and M. Roederer, Polar cap arcs and the open regions, Planet. Space Sci., 31, 193-196, 1983.

Alexeev, I. I. and E. S. BelenkayA, Electric field in open model of the magnetosphere, Geomag. and Aeron., 23, 75-81, 1983a.

Alexeev, I. I. and E. S. BelenkayA, Field-aligned currents in the polar cap, Geomag. and Aeron., 23, 458-464, 1983b.

Alfven, H., Cosmical Electrodynamics, 215 pp., Clarendon Press, Oxford, 1950.

AlfVÉn, H., Electric currents in cosmic plasma, Rev. Geophys. Space Phys., 15, 271-284, 1977.

Alfven, H., Cosmic Plasma, 432 pp., D. Reidel, Dordrecht, 1981.

ARAKI, T., T. KAMEI, and T. IYEMORI, Polar cap vertical currents associated with northward interplanetary magnetic field, Geophys. Res. Lett., 11, 23-26, 1984.

BAKER, K. B. and Y. KAMIDE, A comparison of ionospheric electric fields inferred from STARE drift data and from global IMS magnetometer data, J. Geophys. Res., 90, 1339-1342, 1985.

Bazarzhapov, A. D., M. I. Matveev, and V. M. Mishin, Geomagnetic Variations and Storms, 187 pp., Nauka, Novosibirsk, 1979.

Bazarzhapov, A. D., V. V. Shelomentsev, and V. M. Mishin, Toward choosing a regression model for high-latitude geomagnetic variation field, in Investigations on Geomagnetism, Aeronomy and Solar Physics, No. 66, pp. 138-149, Nauka, Moscow, 1983.

Belov, B. A., R. G. AfoninA, A. E. Levitin, and Y. I. Feldstein, The effects of interplanetary magnetic field orientation on geomagnetic field at northern polar cap, in Variations of Magnetic Field and the Aurora, edited by Y. I. Feldstein and A. E. Levitin, pp. 15-85, IZMIRAN, Moscow, 1977.

BirkelAND, K., Norwegian aurora polaris expedition, 1902-1903, Christiania, part 1, 39-315, 1908; part 2, 319-551, 1913.

Boström, R., A model of the auroral electrojets, J. Geophys. Res., 69, 4983-4994, 1964.

Boström, R., Mechanisms for driving Birkeland currents, in Physics of the Hot Plasma in the Magnetosphere, edited by B. Hultquist and L. Stenflo, pp. 431-447, Plenum, New York, 1975.

Burch, J. L., P. H. Reiff, J. D. Menietti, R. A. Heelis, W. B. Hanson, S. D. Shawhan, E. G. Shelley, M. Suglura, D. R. Weimer, and J. D. Winningham, IMF $B_{y}$-dependent plasma flow and Birkeland currents in the dayside magnetosphere, J. Geophys. Res., 90, 1577-1593, 1985.

Burke, W. J., M. C. Kelley, R. C. Sagalyn, M. Smiddy, and S. T. Lai, Polar cap electric field structures with a northward interplanetary magnetic field, Geophys. Res. Lett., 6, 21-24, 1979.

Burrows, J. R., M. D. Wilson, and I. B. MCDiarmid, Simultaneous field-aligned current and charge particle measurements in the cleft, in Magnetospheric Particles and Fields, edited by B. M. McCormac, pp. 111-123, D. Reidel, Dordrecht, 1976.

Chapman, S., The electric current systems of magnetic storms, Terr. Magn., 40, 349-370, 1935.

Clauer, C. R., P. M. Banks, A. Q. Smith, T. S. Jørgensen, E. Frits-Christensen, S. Vennerstrom, V. 
B. WickWAR, J. D. KELleY, and J. DoupNiK, Observation of interplanetary magnetic field and of ionospheric plasma convection in the vicinity of the dayside polar cleft, Geophys. Res. Lett., 11, 891-894, 1984.

Cowley, S. W. H., Magnetospheric asymmetries associated with the $Y$ component of the IMF, Planet. Space Sci., 29, 79-96, 1981a.

COWLEy, S. W. H., Magnetospheric and ionospheric flow and the interplanetary magnetic field, in Physical Basis of the Ionosphere in the Solar-Terrestrial System, AGARD Conf. Proc., No. 295, Nenilly-SurSeine, France, $1981 \mathrm{~b}$.

Cowley, S. W. H., The causes of convection in the Earth's magnetosphere: a review of developments during the IMS, Rev. Geophys. Space Phys., 20, 531-565, 1982.

Cowley, S. W. H., Solar wind control of magnetospheric convection, in Proc. Conf. Achivements of the IMS, ESA Sp., 483-494, 1984.

Crooker, N. V., Dayside merging and cusp geometry, J. Geophys. Res., 84, 951-959, 1979.

Doyle, M. A. and W. J. Burke, S3-2 measurements of the polar cap potential, J. Geophys. Res., 88, 9125-9133, 1983.

Doyle, M. A., F. J. Rich, W. J. Burke, and M. Smiddy, Field-aligned currents and electric fields observed in the region of the dayside cusp, J. Geophys. Res., 86, 5656-5664, 1981.

Dremuhina, L. A., A. E. Levitin, and Ya. I. Feldstein, About calculations of field-aligned current density at high latitudes by satellite magnetogram. Geomag. and Aeron., 25, 158-160, 1985.

Evans, J. V., J. M. Holt, W. L. Oliver, and R. H. Wand, Millstone Hill incoherent scatter observations of auroral convection over $60^{\circ} \leq \Phi \leq 75^{\circ}, 2$, Initial results, J. Geophys. Res., 85, 41-51, 1980.

FAERMARK, D. S., The method on calculation electric and magnetic fields in high-latitude ionosphere, in Solar wind and magnetospheric phenomena, edited by A. E. Levitin, pp. 122-129, IZMIRAN, Moscow, 1980.

Faermark, D. S., A. L. Krilov, A. E. Levitin, and Ya. I. Feldstein, Restoration from ground-based observations of a three-dimensional current system connected with the interplanetary magnetic field azimuthal component, in Solar Wind and the Magnetosphere, edited by K. G. Ivanov, pp. 47-57, IZMIRAN, Moscow, 1976.

FELDSTEIN, Ya. I., Magnetic field variations in the polar region during magnetically quiet periods and interplanetary magnetic fields, Space Sci. Review, 18, 777-861, 1976.

Feldstein, Ya. I. and A. E. Levitin, Models of conductances in high-latitude ionosphere, Preprint No. 37(511) IZMIRAN, Moscow, 1984.

FeldStein, Ya. I., S. I. ISAEV, and A. I. Lebedinsky, The phenomenology and morphology of auroras, in Annals IQSY, 4, edited by M. Dogan and S. Roukan, paper 20, pp. 311-348, M. I. T. Press, Boston, 1969.

Feldstein, Ya. I., P. V. Sumaruk, and N. F. Shevnina, To the diagnostics of the azimuthal component of the interplanetary magnetic field, C. R. Acad. Sci. USSR, 222, 833-836, 1975.

Feldstein, Ya. I., A. E. Levitin, R. G. AfoninA, and B. A. Belov, Magnetosphere-ionosphere coupling, in Interplanetary medium and Earth's magnetosphere, edited by Ya. I. Feldstein and A. E. Levitin, pp. 64-115, Nauka, Moscow, 1982a.

Feldstein, Ya. I., A. E. Levitin, and D. S. Faermark, Large-scale electric fields and electrostatic potentials in the high-latitude ionosphere under various situations in the interplanetary medium, Proceedings V Stara Zagora Zeminar, Bulgaria, Magnetosphere-ionosphere processes and airglow, 4-75, 1982b.

Feldstein, Ya. I., R. G. Afonina, B. A. Belov, and A. E. Levitin, Magnetic field and field-aligned current variations in the polar cusp controlled by interplanetary medium parameters, Planet. Space Sci., 30, 635-640, 1982c.

Feldstein, Ya. I., A. E. Levitin, D. S. Faermark, R. G. Afonina, and B. A. Belov, Electric fields and potential patterns in the high-latitude ionosphere for different situations in interplanetary space, Planet. Space Sci., 32, 907-923, 1984a.

Feldstein, Ya. I., A. E. Levitin, and D. S. Faermark, Large-scale electric field in the high-latitude ionosphere, in Polar Ionosphere and Magnetosphere-Ionosphere Interaction, pp. 91-129, Yakutsk, $1984 \mathrm{~b}$.

FOSTER, J. C., An empirical electric field model derived from Chatanika radar data, J. Geophys. Res., 88, 981-987, 1983.

FOSTER, J. C., Ionospheric signatures of magnetospheric convection, J. Geophys. Res., 89, 855-865, 1984. 
Foster, J. C., J. R. DoupNiK, and G. S. StILES, Large-scale patterns of auroral ionospheric convection observed with the Chatanika radar, J. Geophys. Res., 86, 11357-11371, 1981.

Foster, J. C., J. M. Holt, J. D. Kelly, and V. B. Wickwar, High-resolution observations of electric fields and $F$-region plasma parameters in the cleft ionosphere, in The Polar Cusp, edited by J. A. Holtet and A. Egeland, pp. 349-364, D. Reidel, Dordrecht, 1985.

FriIS-ChristensEn, E., The effect of the IMF on convection patterns and equivalent currents in the polar cap and cusp, in Magnetospheric Study 1979, pp. 290-295, Japanese IMS Committee, 1979.

FriIS-Christensen, E., High latitude ionospheric currents, in Exploration of the Polar Upper Atmosphere, edited by C. S. Deehr and J. A. Holtet, pp. 315-328, D. Reidel, Dordrecht, 1981.

Friss-Christensen, E. and J. Wilhjelm, Polar currents for different directions of the interplanetary magnetic field in the $Y$-Z plane, J. Geophys. Res., 80, 1248-1260, 1975.

Frits-Christensen, E., K. Lassen, J. Wilhjelm, J. M. Wilcox, W. Gonzalez, and D. S. Colburn, Critical component of the interplanetary magnetic field responsible for large geomagnetic effects in the polar cap, J. Geophys. Res., 77, 3371-3380, 1972.

Frits-Christensen, E., Y. Kamide, A. D. Richmond, and S. Matsushita, Interplanetary magnetic field control of high-latitude electric fields and currents determined from Greenland magnetometer data, $J$. Geophys. Res., 90, 1325-1338, 1985.

Fukushima, N., Generalized theorem for no ground magnetic effect of vertical currents connected with Pedersen currents in the uniform-conductivity ionosphere, Rept. Ionosph. Space Res. Japan, 30, 35-40, 1976.

Galperin, Yu. I., N. V. Ponomarev, and A. G. Zosimova, Equatorial ionospheric anomaly and interplanetary magnetic field, J. Geophys. Res., 83, 4265-4272, 1978.

Gurevich, A. V., A. L. Krylov, and E. E. Tsedilina, Electric fields in the Earth's magnetosphere and ionosphere, in Investigations on Geomagnetism, Aeronomy and Solar Physics, No. 35, pp. 113-160, Nauka, Moscow, 1975.

Gurevich, A. V., A. L. Krylov, and E. E. Tsedilina, Electric fields in the Earth's magnetosphere and ionosphere, Space Sci. Rev., 19, 59-116, 1976.

Harel, M., R. A. Wolf, P. H. Reiff, R. W. SPiro, W. J. Burke, F. J. Rich, and M. Smiddy, Quantitative simulation of a magnetospheric substorm, 1. Model logic and overview, J. Geophys. Res., 86, 2217-2241, 1981.

HEELIS, R. A., The effects of interplanetary magnetic field orientation on dayside high-latitude ionospheric convection, J. Geophys. Res., 89, 2873-2880, 1984.

Heelis, R. A., W. B. HAnson, and J. L. BuRCh, Ion convection velocity reversals in the dayside cleft, $J$. Geophys. Res., 81, 3803-3809, 1976.

HePPNER, J. P., Polar cap electric field directions related to the interplanetary magnetic field direction, $J$. Geophys. Res., 77, 4877-4889, 1972.

HePPner, J. P., Empirical models of high-latitude electric fields, J. Geophys. Res., 82, 1115-1125, 1977.

HePPNER, J. P., J. D. STOLARIK, and E. M. WESCOTT, Electric field measurements and the identification of currents causing magnetic disturbances in the polar cap, J. Geophys. Res., 76, 6028-6041, 1971.

IIJIMA, T., Field-aligned currents during northward IMF, in Magnetospheric Currents, edited by T. A. Potemra, pp. 115-122, AGU, Washington, 1984.

Iijima, T. and T. A. Potemra, The amplitude distribution of field-aligned currents at northern high latitudes observed by TRIAD, J. Geophys. Res., 81, 2165-2174, 1976a.

IIJIMA, T. and T. A. PotemRA, Field-aligned currents in the dayside cusp observed by TRIAD, J. Geophys. Res., 81, 5971-5979, 1976b.

IiJima, T. and T. A. Potemra, The relationship between interplanetary quantities and Birkeland current densities, Geophys. Res. Lett., 9, 442-445, 1982.

Iijima, T., R. Fujil, T. A. Potemra, and N. A. Saflekos, Field-aligned currents in the south polar cusp and their relationship to the interplanetary magnetic field, J. Geophys. Res., 83, 5595-5603, 1978.

injma, T., T. A. Potemra, L. J. Zanetti, and P. F. Bythrow, Large-scale Birkeland currents in the dayside polar region during strongly northward IMF: a new Birkeland current system, J. Geophys. Res., 89, 7441-7452, 1984.

Ivanov, K. G. and L.V. Evdokimova, Electric field of polar caps presuming a magnetopause to be an anizotropic rotating discontinuity, Geomag. and Aeron., 15, 303-307, 1975.

IWASAKI, N., Localized abnormal geomagnetic disturbance near the geomagnetic pole and simultaneous ionospheric variation, Rep. Ionosph. Space Res. Japan, 25, 163-186, 1971. 
JAGGI, R. V. and R. A. Wolf, Self-consistent calculation of the motion of a sheet of ions in the magnetosphere, J. Geophys. Res., 78, 2852-2866, 1973.

JøRGENSEN, T. S., Electric field in the dayside auroral region, in Exploration of the Polar Upper Atmosphere, edited by C. S. Deehr and J. A. Holtet, pp. 267-280, D. Reidel, Dordrecht, 1981.

Jørgensen, T. S., E. Frils-Christensen, V. B. Wickwar, J. D. Kelly, C. R. Clauer, and P. M. Banks, On the reversal from "sunward" to "antisunward" plasma convection in the dayside high latitude ionosphere, Geophys. Res. Lett., 11, 887-890, 1984.

KAMIDE, Y., The relationship between field-aligned currents and the auroral electrojets: a review, Space Sci. Rev., 31, 127-245, 1982.

Kamide, Y., IMS advances in studies of field-aligned currents and their related electrodynamics, in Proc. Conf. Achievements of the IMS, ESA SP, pp. 243-256, 1984.

KAmide, Y. and H. W. KROEHL, Computer simulations of ionospheric electric fields and currents. Final report of the IMS magnetometer project, The Bulletin of the Institute of Computer Sciences, Kyoto Sangyo University, 2, N2, 1-208, 1984.

KAMIDE, Y. and S. MATSUSHITA, Simulation studies of ionospheric electric fields and currents in relation to field-aligned currents. 1. Quiet periods, J. Geophys. Res., 84, 4083-4098, 1979.

KAmide, Y. and A. D. Richmond, Ionospheric conductivity dependence of electric fields and currents estimated from ground magnetic observations, J. Geophys. Res., 87, 8331-8337, 1982.

Kamide, Y., A. D. Richmond, and S. Matsushita, Estimations of ionospheric electric fields, ionospheric currents and field-aligned currents from ground magnetic records, J. Geophys. Res., 86, 801-813, 1981.

Kamide, Y., R. A. Wolf, R. W. Spiro, and A. D. Richmond, Inferring electric fields and currents from ground-magnetometer data: a test with theoretically derived inputs, EOS, 63, 420, 1982.

Kamide, Y., H. W. Kroehl, B. A. Hausman, R. L. McPherron, S. I. Akasofu, A. D. Richmond, P. H. ReifF, and S. MAtSushita, Numerical modeling of ionospheric parameters from global IMS magnetometer data for the CDAW-6 intervals, WDCA for Solar-Terrestrial Physics, Report UAG$88,1983$.

KawASAKI, K., A model of the electric field over the polar cap, Rept. Ionosph. Space Res. Japan, 29, $157-161,1975$.

Kawasaki, K., F. Yasuhara, and S.-I. Akasofu, Short-period interplanetary and polar magnetic field variations, Planet. Space Sci., 21, 1743-1755, 1973.

KERN, J. W., Analysis of polar magnetic storms, J. Geomag. Geoelectr., 18, 125-131, 1966.

KIRK PATRICK, C. B., On current systems proposed for $S D$ in theory of magnetic storms, J. Geophys. Res., 57, 511-526, 1952.

KiSABETH, J. L., On calculating magnetic and vector potential fields due to large scale magnetospheric current systems and induced currents in an infinitely conducting earth, in Quantitative Modeling of Magnetospheric Processes, edited by W. Olson, pp. 473-498, Amer. Geophys. Union, Geophys. Monograph No. 21, Washington, 1979.

KisAbeth, J. L. and G. Rostoker, Modelling of three-dimensional current systems associated with magnetospheric substorms, Geophys. J. Roy. Astron Soc., 49, 655-683, 1977.

Krylov, A., Mathematical models of electric fields and currents in the Earth's magnetosphere. D. S. Thesis, Moscow, 1974.

KuZnetsov, B. M. and O. A. Troshichev, On the nature of polar cap magnetic activity during undisturbed periods, Planet. Space Sci., 25, 15-21, 1977.

LEONTYEV, S. V. and W. B. LyATSKY, Electric field and currents connected with $Y$-component of interplanetary magnetic field, Planet. Space Sci., 22, 811-819, 1974.

Levitin, A. E., B. A. Belov, R. G. Afonina, D. S. Faermark, and Ya. I. Feldstein, Three-dimensional current systems in the north polar cap for different types of geomagnetic field variations, in Variations of Geomagnetic Field and the Aurora, edited by Ya. I. Feldstein, pp. 86-106, IZMIRAN, Moscow, 1977 .

Levitin, A. E., B. A. Belov, R. G. Afonina, D. S. Faermark, and Ya. I. Feldstein, Reverse polarity of field-aligned currents and its interpretation, in Variations of Geomagnetic Field and Aurora, edited by Ya. I. Feldstein, pp. 107-111, IZMIRAN, Moscow, 1977 b.

Levitin, A. E., R. G. Afonina, B. A. Belov, and Ya. I. Feldstein, Geomagnetic variations and field-aligned currents at northern high-latitudes and their relations to solar wnd parameters, Phil. Trans. R. Soc., A304, 253-301, 1982. 
Levitin, A. E., Ya. I. Feldstein, R. G. Afonina, B. A. Belov, L. A. Dremuhina, D. S. Faermark, Yu. Z. Demidova, M. Yu. Markova, S. I. Avdjushin, V. V. Mihnevich, P. M. SvidskiY, and V. Yu. GaIDukov, The Model Large-Scale Electric Field and Currents in High-Latitude Ionosphere, parts. 1-6, Gidrometeoizdat, Moscow, 1984.

LyATSKY, V. B., The Current Systems of Magnetospheric-Ionospheric Disturbances, 196 pp., Nauka, Leningrad, 1978.

LyATSKy, V. B. and Yu. P. Maltsev, Three-dimensional current system of the magnetic substorm, in Geophysical Research in Auroral Zone, edited by S. I. Isaev, pp. 74-86, Polar Geophysical Institute, Apatity, 1972.

LyATSKy, V. B. and Yu. P. MALtSev, The Magnetosphere-Ionosphere Interaction, 188 pp., Nauka, Moscow, 1983.

Maezawa, K., Magnetospheric convection induced by the positive and negative Z-components of the interplanetary magnetic field: quantitative analysis using polar cap magnetic records, J. Geophys. Res., 81, 2289-2303, 1976.

Mansurov, S. M., New evidence of the relationship between magnetic field in space and on the earth, Geomag. and Aeron., 9, 768-773, 1969.

Matveev, M. I. and G. V. ShPynev, Determination of electric fields and field-aligned currents in the magnetosphere on data of geomagnetic variations (high-latitude region), in Investigations on Geomagnetism, Aeronomy and Solar Physics, No. 36, pp. 34-39, Nauka, Moscow, 1975.

McDiarmid, I. B., E. E. Budzinski, M. D. Wilson, and J. R. Burrows, Reverse polarity field-aligned currents at high latitudes, J. Geophys. Res., 82, 1513-1519, 1977.

McDiarmid, I. B., J. R. Burrows, and M. D. Wilson, Comparison of magnetic field perturbations at high-latitudes with charged particle and IMF measurements, J. Geophys. Res., 83, 681-688, 1978.

MCDiarmid, I. B., J. R. Burrows, and M. D. Wilson, Large-scale magnetic field perturbations and particle measurments at $1400 \mathrm{~km}$ on the dayside, J. Geophys. Res., 84, 1431-1441, 1979.

McDiarmid, I. B., J. R. Burrows, and M. D. Wilson, Comparison of magnetic field perturbations and solar electron profiles in the polar cap, J. Geophys. Res., 85, 1163-1170, 1980.

Metha, N. C., Ionospheric electrodynamics and its coupling to the magnetosphere, Ph. D. Thesis, Univ. of Calif., San Diego, 1979.

Mishin, V. M., Magnetospheric and magnetic substorms (review), Geomag. and Aeron., 18, 961-991, 1978.

Mishin, V. M. and G. V. Popov, About the field-aligned currents in the magnetosphere, in Invest. on Geomagnetism, Aeronomie, and Solar Physics, No. 8, edited by V. M. Mishin, pp. 3-28, Irkutsk, 1969.

Mishin, V. M., M. I. Matveev, G. B. ShPynev, A. D. Bazarzhapov, and V. K. Kompanetz, Preliminary results of calculations of three-dimensional electric current system in the magnetosphere using ground-based data of geomagnetic disturbances, in Investigations on Geomagnetism, Aeronomy and Solar Physics, No. 43, pp. 14-17, Nauka, Moscow, 1977.

Mishin, V. M., A. D. Bazarzhapov, A. A. Anistratenko, and L. V. Aksenova, Electric currents and magnetospheric convection caused by a non-magnetized solar wind, Geomag. and Aeron., 18, 751-753, 1978.

Mishin, V. M., A. D. Bazarzhapov, and G. B. Shpynev, Electric fields and currents in the earth's magnetosphere, in Dynamics of the Magnetosphere, edited by S.-I. Akasofu, pp. 249-268, D. Reidel, Dordrecht, 1980.

Mishin, V. M., G. B. ShPynev, and A. D. Bazarzhapov, Large-scale electric field and currents in the high-latitude ionosphere and magnetosphere as a function of solar wind parameters, Adv. Space Res., 1, 159-169, 1981.

Mishin, V. M., G. B. ShPynev, and A. D. Bazarzhapov, Computation of electric field and currents in the earth's magnetosphere on a continuous basis by means of ground geomagnetic measurements, in Investigations on Geomagnetism, Aeronomy and Solar Physics, No. 58, pp. 178-186, Nauka, Moscow, 1982.

Mishin, V. M., T. I. Saifudinova, D. Sh. Shirapov, S. B. Lunyushin, and V. V. Shelomentsev, The analysis of the CDAW-6 substorms of March 22, 1979, in Investigations on Geomagnetism, Aeronomy and Solar Physics, pp. 151-200, Nauka, Moscow, 1984.

Mozer, F.S., Electric field evidence on the viscous interaction at the magnetopause, Geophys. Res. Lett., 11, 135-138, 1984. 
Mozer, F. C., C. A. Cattell, M. K. Hudson, R. L. Lysak, M. Temerin, and R. B. Torbert, Satellite measurements and theories of low altitude auroral particle acceleration, Space Sci. Rev., 27, 155-213, 1980.

NishidA, A., Geomagnetic DP2 fluctuations and associated magnetospheric phenomena, J. Geophys. Res., 73, 1795-1803, 1968a.

NishidA, A., Coherence of geomagnetic DP2 fluctuations with interplanetary magnetic variations, $J$. Geophys. Res., 73, 5549-5559, 1968 b.

NishidA, A., Geomagnetic Diagnosis of the Magnetosphere, Springer Verlag, New York-Heidelberg, 1978.

NishidA, A., IMF control on the Earth's magnetosphere, Space Sci. Reviews, 34, 185-200, 1983.

Potemra, T. A., Large-scale characteristics of field-aligned currents determined from the TRIAD magnetometer experiment, in Dynamical and Chemical Coupling, edited by B. Grandel and J. A. Holtet, pp. 337-352, D. Reidel, Dordrecht, 1977.

Potemra, T. A., Birkeland currents: present understanding and some remaining questions, in HighLatitude Space Plasma Physics, edited by B. Hultqvist and T. Hagfors, pp. 335-353, Plenum, New York, 1983.

Potemra, T. A., T. Iijima, and N. A. Saflekos, Large-scale characteristics of Birkeland currents, in Dynamics of the Magnetosphere, edited by S.-I. Akasofu, pp. 165-199, D. Reidel, Dordrecht, 1980.

Potemra, T. A., L. J. Zanetti, P. F. Bythrow, A. T. Y. Lul, and T. IiJima, $\boldsymbol{B}_{y}$-dependent convection patterns during northward interplanetary magnetic field, J. Geophys. Res., 89, 9753-9760, 1984.

Primdahl, F. and F. SPANgSlev, Cross-polar cap horizontal $E$ region currents related to magnetic disturbances and to measured electric fields, J. Geophys. Res., 82, 1137-1143, 1977.

Primdahl, F., J. K. Walker, F. Spangslev, J. K. Olesen, U. Fahleson, and E. Ungstrup, Sunlit cleft and polar cap ionospheric currents determined from rocket-borne magnetic field, plasma and electric field observations, J. Geophys. Res., 84, 6458-6470, 1979.

Pudovkin, M. I. and S. A. Zaitseva, Electric field in the polar cap, Geomag. and Aeron., 23, 285-289, 1983.

Pudovkin, M. I., S. A. Zaitseva, and V. F. Zakharov, Structure of the magnetosphere and magnetosphere-ionosphere interaction, in Polar Ionosphere and Magnetosphere-Ionosphere Interaction, pp. 3-49, Yakutsk, 1984.

ReIFF, P. H., Models of auroral-zone conductances, in Magnetospheric Currents, edited by T. A. Potemra, pp. 180-191, Geophys. Monograph. No. 28, AGU, Washington, 1984.

ReIFF, P. H. and J. L. BuRCH, IMF $B_{y}$-dependent plasma flow and Birkeland currents in the dayside magnetosphere: 2. A global model for northward and southward IMF, J. Geophys. Res., 90, 1595-1609, 1985.

ReifF, P. H., R. W. SPIRo, and T. W. HiLl, Dependence of polar cap potential drop on interplanetary parameters, J. Geophys. Res., 86, 7639-7648, 1981.

Rezhenov, B. V., Convection at high-latitudes when the interplanetary magnetic field is northward, Planet. Space Sci., 29, 687-693, 1981.

Rezhenov, B. V., Lyatsky, V. B., and Yu. P. Maltsev, Convection in the polar cap after the interplanetary magnetic field becomes northward, Planet. Space Sci., 28, 595-597, 1980.

Robinson, R. M. and R. R. VONDRAK, Measurements of $E$ region ionization and conductivity produced by solar illumination at high latitudes, J. Geophys. Res., 89, 3951-3956, 1984.

Saflekos, N. A. and T. A. Potemra, The orientation of Birkeland currents sheets in the dayside polar region and its relationship to the IMF, J. Geophys. Res., 85, 1987-1994, 1980.

Saflekos, N. A., T. A. Potemra, and T. IIJima, Small-scale transverse magnetic field disturbances in the polar regions observed by TRIAD, J. Geophys. Res., 83, 1493-1502, 1978.

Saflekos, N. A., R. E. Sheehan, and R. L. Carovillano, Global nature of field-aligned currents and their relation to auroral phenomena, Rev. Geophys. Space Physics, 20, 709-734, 1982.

Sergeev, V. A. and B. M. KuZnetsov, Quantitative dependence of the polar cap electric field on the IMF $B_{z}$-component and solar wind velocity, Planet. Space Sci., 29, 205-213, 1981.

Sergeev, V. A. and N. A. Tsyganenko, The Earth's Magnetosphere, edited by M. I. Pudovkin, 279 pp., Nauka, Moscow, 1980.

Spiro, R. W., P. H. Reiff, and L. J. MAHER, Precipitating electron energy flux and auroral zone conductances: an empirical model, J. Geophys. Res., 87, 8215-8227, 1982.

Stern, D. P., A study of the electric field in an open magnetospheric model, J. Geophys. Res., 78, 7292-7305, 1973. 
Stern, D. P., Large-scale electric fields in the Earth's magnetosphere, Rev. Geophys. Space Phys., 15, 156-194, 1977.

Stern, D. P., The origin of Birkeland currents, Rev. Geophys. Space Phys., 21, 125-138, 1983.

Sugiura, M., Identification of the polar cap boundary and the auroral belt in the high-latitude magnetosphere: a model for field-aligned currents, J. Geophys. Res., 80, 2057-2068, 1975.

Sumaruk, P. V. and Ya. I. FeldStein, Sector structure of the interplanetary magnetic field and magnetic disturbances in nearpole region, Kosmich. Issled. (Space Invest.), 11, 155-160, 1973.

SvalgaARD, L., Sector structure of the interplanetary magnetic field and daily variation of the geomagnetic field at high latitudes, Geophys. Papers R-6, Danish Meteorol. Inst., 1968.

Troshichev, O. A., Polar magnetic disturbances and field-aligned currents, Space Sci. Rev., 32, 275-360, 1982.

Troshichev, O. A., Solar wind control of electric fields and ionosphere, in Achievements of the International Magnetospheric Study(IMS), Proc. Int. Symp. Graz, Austria, 26-28 June, 1984, Europ. pp. 407-416, Space Agency, 1984.

Troshichev, O. A. and V. A. Gizler, Model of the field-aligned currents in dayside cleft depending on interplanetary magnetic field orientation, Geomagnetic Researches, edited by M. I. Pudovkin, No. 27, pp. 108-110, Soviet Radio, Moscow, 1980.

Troshichev, O. A. and N. A. Tsyganenko, Correlation ratios between variations of interplanetary magnetic field and magnetic disturbances in the polar cap, in Geomagnetic Researches, No. 25, pp. 47-59, Soviet Radio, Moscow, 1979.

Troshichev, O. A., V. P. Vasiliev, and B. M. Kuznetsov, Peculiarities of magnetic disturbances in winter and summer polar cap, in Geomagnitnjie Issledovaniya, No. 26, pp. 62-71, Soviet Radio, Moscow, 1979.

TVERSKoy, B. A., Electric fields in the magnetosphere and the origin of trapped radiation, in Solar-Terr. Phys., 1970, edited by E. R. Dyer, part. 3, pp. 297-317, D. Reidel, Dordrecht, 1972.

VAsyliunas, V. M., Mathematical models of magnetospheric convection and its coupling to the ionosphere, in Particles and Fields in the Magnetosphere, edited by B. M. McCormac, pp. 60-71, D. Reidel, Dordrecht, 1970.

Vasyliunas, V. M., The interrelationship of magnetospheric processes, in Earth's Magnetospheric Processes, edited by B. M. McCormac, pp. 29-48, D. Reidel, Dordrecht, 1972.

Vennerstrom, S., E. Fris-Christensen, T. S. Jørgensen, O. Rasmussen, C. R. Clauer, and V. B. WICKWAR, Ionospheric currents and F-region plasma boundaries near the dayside cusp, Geophys. Res. Lett., 11, 903-906, 1984.

Vestine, E. H. and S. ChAPMAN, The electric current-system of geomagnetic disturbance, Terr. Magn., 43, 351-382, 1938.

Volland, H., Models of global electric fields within the magnetosphere, Ann. Geophys., 31, 154-173, 1975a.

Volland, H., Differential rotation of the magnetospheric plasma as cause of the Svalgaard-Mansurov effect, J. Geophys. Res., 80, 2311-2315, 1975b.

Wallis, D. D. and E. E. Budzinski, Empirical models of height integrated conductivities, J. Geophys. Res., 86, 125-137, 1981.

Wilhjelm, J., E. Frils-Christensen, and T. A. Potemra, The relationship between ionospheric and field-aligned currents in the dayside cusp, J. Geophys. Res., 83, 5586-5594, 1978.

WOLF, R. A. and Y. KAMIDE, Inferring electric fields and currents from ground-magnetometer data: a test with theoretically derived inputs, J. Geophys. Res., 88, 8129-8135, 1983.

Wolf, R. A. and R. W. SPIRo, Ionosphere-magnetosphere coupling and convections, in Proc. Conf. Achievements of the IMS, ESA SP, pp. 417-426, 1984.

Wygant, J. R., R. B. TorberT, and F. S. MOZer, Comparison of S3-3 polar cap potential drops with the interplanetary magnetic field and models of magnetopause reconnection, J. Geophys. Res., 88, 5727-5735, 1983.

Yasuhara, F., Greenwald, and S.-I. AKasofu, On the rotation of the polar cap potential pattern and associated polar phenomena, J. Geophys. Res., 88, 5773-5777, 1983.

Zmuda, A. J. and J. C. Armstrong, The diurnal flow pattern of field-aligned currents, J. Geophys. Res., 79, 4611-4619, 1974. 